\title{
ERGODIC THEORY, GROUP REPRESENTATIONS, AND RIGIDITY
}

\author{
BY ROBERT J. ZIMMER
}

\section{CONTENTS}

1. Basic Notions

2. Ergodicity theorems

3. Cocycles

4. Generalized discrete spectrum

5. Amenability

6. Rigidity: The Mostow-Margulis theorem and a generalization to ergodic actions

7. Complements to the rigidity theorem for ergodic actions: Foliations by symmetric spaces and Kazhdan's property (T)

8. Margulis' finiteness theorem

9. Margulis' arithmeticity theorem

These notes represent a mildly expanded version of lectures delivered at the C.I.M.E. summer session on harmonic analysis and group representations in Cortona, Italy, June-July 1980. The author would like to express his thanks and appreciation to the organizers of the conference, Michael Cowling, Sandro Figà-Talamanca, and Massimo Picardello, for inviting him to deliver these lectures and for their most warm and generous hospitality during his stay in Italy. We would also like to thank the other participants of the conference for their interest in these lectures. Finally, we would like to thank Terese S. Zimmer for (among innumerable other things that we need not go into here) helping with the translation of [29].

ADDED IN PROOF. Complete and detailed proofs of all the results discussed in these lectures (except those in section 4), as well as further developments, will appear in a forthcoming monograph of the author.

1. Basic notions. In these lectures we discuss some topics concerning the relationship of ergodic theory, representation theory, and the structure of Lie groups and their discrete subgroups.

In studying the representation theory of groups, the assumption of compactness on the group essentially allows one to reduce to a finite dimensional situation, in which case one often can obtain complete information. For noncompact groups, of course, no such reduction is possible and the situation is much more complex. When studying general actions of groups, a somewhat

Received by the editors November 15, 1981.

1980 Mathematics Subject Classification. Primary 22D10, 22D40, 22E40, 28D15, 53C35, 57R30, $57 \mathrm{~S} 20$.

${ }^{1}$ Partially supported by a Sloan Foundation Fellowship and NSF Grant MCS 79-05036. 
similar situation arises. In the compact case every orbit will be closed, the space of orbits will have a reasonable structure, and one can often find nice (with respect to the action) neighborhoods of orbits. A large amount of information about actions of finite and compact groups has been obtained by topological methods. However, once again, if the compactness assumption on the group is dropped, one faces many additional problems. In particular, one can have orbits which are dense (for example, the irrational flow on the torus) and the orbit space may be so badly behaved as to have no continuous functions but constants. Furthermore, moving from a point to a nearby point may produce an orbit which does not follow closely to the original orbit. If one wishes to deal with actions in the noncompact case, this phenomenon of complicated orbit structure must be faced. For many actions, e.g., differentiable actions on manifolds, there are natural measures that behave well with respect to the action. A significant part of ergodic theory is the study of group actions on measure spaces. In particular, ergodic theory aims to understand the phenomenon of bad orbit structure in the presence of a measure.

Throughout these lectures, $G$ will be a locally compact, second countable group. Let $(S, \mu)$ be a standard measure space, and assume we have an action $S \times G \rightarrow S$ which is a Borel function. Then $\mu$ (which is always assumed to be $\sigma$-finite) is invariant if $\mu(A g)=\mu(A)$ for all $A \subset S$ and $g \in G$, and quasiinvariant if $\mu(A g)=0$ if and only if $\mu(A)=0$.

Definition 1.1. The action is called ergodic if $A \subset S$ is $G$-invariant implies $\mu(A)=0$ or $\mu(S-A)=0$.

Clearly any transitive action is ergodic, or, more generally, any essentially transitive action (i.e., transitive on the complement of a null set). We can then write $S=G / G_{0}$ where $G_{0} \subset G$ is a closed subgroup. An ergodic action that is not essentially transitive will be called properly ergodic.

EXAMPLE 1.2. Let $S=\{z \in \mathbf{C}|| z \mid=1\}$ and $T: S \rightarrow S$ be $T(z)=e^{i \alpha} z$ where $\alpha / 2 \pi$ is irrational. Then $T$ generates a $Z$-action. If $A \subset S$ is invariant, let $\chi_{A}(z)=\sum a_{n} z^{n}$ be the $L^{2}$-Fourier expansion of its characteristic function. Then by invariance $\chi_{A}(z)=\chi_{A}\left(e^{i \alpha} z\right)=\sum a_{n} e^{i n \alpha} z^{n}$. Thus $a_{n} e^{i n \alpha}=a_{n}$ and so $a_{n}=0$ for $n \neq 0$. This implies $\chi_{A}$ is constant, so the action is properly ergodic.

REMARK. If $S$ is a (second countable) topological space and $\mu$ is positive on open sets, then proper ergodicity implies almost every orbit is a dense null set. This is one sense in which proper ergodicity is a reflection of complicated orbits. Another is the following.

Proposition 1.3 [12]. Let $G$ act continuously on $S$ where $S$ is metrizable by a complete separable metric. Then the following are equivalent. (We say the action is "smooth" if they hold.)

(i) Every G-orbit is locally closed.

(ii) $S / G$ is $T_{0}$ in the quotient topology.

(iii) The quotient Borel structure on $S / G$ is countably separated and generated (i.e., there is a countable family $\left\{A_{i}\right\}$ separating points and generating the Borel structure).

(iv) Every quasi-invariant ergodic measure is supported on an orbit. 
Proof. (i) $\Rightarrow$ (ii) $\Rightarrow$ (iii) are elementary. To see (iii) $\Rightarrow$ (iv), let $p: S \rightarrow S / G$ be the projection, and $\mu$ an ergodic probability measure on $S$. Then $\nu=p_{*}(\mu)$ is a measure on $S / G$ with the property that for any Borel set $B \subset S / G, \nu(B)=0$ or 1 . Since $S / G$ is countably separated and generated, $\nu$ is supported on a point, so $\mu$ is supported on an orbit. The implication (iv) $\Rightarrow$ (i) is difficult (and we will not be using it).

We will be making constant use of the implication (i) $\Rightarrow$ (iv). For example,

COROLlARY 1.4. Every ergodic action of a compact group is essentially transitive.

If the action is on a metric space, this follows immediately. However, a theorem of Varadarajan [45] implies that any action can be so realized.

COROLlARY 1.5. Every ergodic algebraic action of a real (or p-adic) algebraic group (more precisely, the real or p-adic points) on an algebraic variety is essentially transitive.

This follows from the theorem of Borel and Borel-Serre that orbits are locally closed $[3,6]$.

While the decomposition of a general action into orbits may not be satisfactory there is always a good decomposition into ergodic components.

Proposition 1.6. Let $(S, \mu)$ be a G-space. Then there is a standard measure space $(E, \nu)$, a conull G-invariant set $S_{0} \subset S$, and a G-invariant Borel map $\varphi$ : $S \rightarrow E$ with $\varphi_{*}(\mu)=\nu$ such that, writing $\mu=\int^{\oplus} \mu_{y} d \nu(y)$ where $\mu_{y}$ is supported on $\varphi^{-1}(y)$, we have $\mu_{y}$ is quasi-invariant and ergodic under $G$ for almost all $y$.

$(E, \nu)$ is called the space of ergodic components of the action (and is essentially uniquely determined by the above conditions).

We now discuss some notions of "isomorphism".

Definition 1.7. Let $(S, \mu),\left(S^{\prime}, \mu^{\prime}\right)$ be ergodic $G$-spaces. Call them conjugate if modulo null sets there is $\varphi: S \rightarrow S^{\prime}$ with

(i) $\varphi$ a bijective Borel isomorphism.

(ii) $\varphi_{*}(\mu) \sim \mu^{\prime}$ (i.e., same null sets).

(iii) $\varphi(s g)=\varphi(s) g$.

If $A \in \operatorname{Aut}(G)$ and $S$ is a $G$-space, we have a new $G$-action on $S$ by defining $s \circ g=s \cdot A(g)$.

DEFINITION 1.8. Call two actions automorphically conjugate if they become conjugate when modified by some automorphism.

An a priori much weaker notion is simply to ask for the orbit pictures to be the same. Here, we can compare actions of different groups.

Definition 1.9. Suppose $(S, \mu)$ is a $G$-space, $\left(S^{\prime}, \mu^{\prime}\right)$ a $G^{\prime}$-space. Call the actions orbit equivalent if (modulo null sets) there exists $\varphi: S \rightarrow S^{\prime}$ with

(i) $\varphi$ a bijective Borel isomorphism.

(ii) $\varphi_{*}(\mu) \sim \mu^{\prime}$.

(iii) $\varphi(G$-orbit $)=G^{\prime}$-orbit.

If $\varphi:(X, \mu) \rightarrow(Y, \nu)$ is a measure class preserving $G$-map of $G$-spaces we call $X$ an extension of $Y$ or $Y$ a factor of $X$. Observe that we automatically 
have $\nu(Y-\varphi(X))=0$. If $H \subset G$ is a subgroup, and $X$ is an ergodic $G$-space, we can restrict to obtain an action of $H$, which of course no longer need be ergodic. In the other direction, we can induce. Namely, suppose $S$ is an ergodic $H$-space and $H \subset G$ is a closed subgroup. Then we obtain a naturally associated $G$-space as follows. Let $H$ act on $S \times G$ by $(s, g) h=(s h, g h)$ and let $X=(S \times G) / H$. Then $G$ acts on $S \times G$ by $(s, g) \tilde{g}=\left(s, \tilde{g}^{-1} g\right)$, and this action commutes with the $H$-action. Hence there is an induced action of $G$ on $X$ which will be ergodic with its natural measure class.

Definition 1.10. $X$ is called the ergodic $G$-space induced from the $G$-action, and we denote it by $\operatorname{ind}_{H}^{G}(S)$.

For example, if $H=Z, G=\mathbf{R}$, then $X$ can be identified with $(S \times[0,1]) / \sim$ where $\sim$ identifies $(s, 1)$ with $(T s, 0)$. Under the induced R-action a point simply flows up along the line it is in with unit speed.

Given an ergodic $G$-space $X$, it is useful to know when it is induced from an action of a subgroup. The following is helpful in this regard.

Proposition 1.11 [52]. If $X$ is an ergodic $G$-space and $H \subset G$ is a closed subgroup, then $X=\operatorname{ind}_{H}^{G}(S)$ for some $H$-space $S$ if and only if $G / H$ is a factor of $X$, i.e., there is a measure class preserving $G$-map $X \rightarrow G / H$.

If $X$ is a $G$-space, is there a unique (up to conjugacy) smallest closed subgroup from which it is induced? The answer in general is no, but we have the following

Proposition 1.12 [54]. Suppose $G$ is (the real points, or $k$-points, $k$ a p-adic field) of an algebraic group and $X$ an ergodic $G$-space. Then there is a unique conjugacy class of algebraic subgroups such that $X=\operatorname{ind}_{H}^{G}(S)$ for $H$ algebraic (and some $S$ ), if and only if $H$ contains a member of this conjugacy class.

Definition 1.13 [54]. If $H$ is in this class, call $H$ the algebraic hull of the action. If this is all of $G$, call the action Zariski dense.

If $X=G / G_{0}$, then the algebraic hull is just usual algebraic hull of the group $G_{0}$.

Proof of 1.12. There exist minimal such groups from the descending chain condition on algebraic subgroups. Suppose $H_{1}, H_{2} \subset G$ are two such minimal algebraic groups. We have $\varphi_{i}: X \rightarrow G / H_{i}$. Let $\varphi=\left(\varphi_{1}, \varphi_{2}\right): X \rightarrow G / H_{1} \times$ $G / H_{2}$. Then $\varphi_{*}(\mu)$ is an ergodic quasi-invariant measure on $G / H_{1} \times G / H_{2}$. But the $G$-action on this product is algebraic, so $\varphi_{*}(\mu)$ is supported on an orbit. But as a $G$-space, an orbit is $G /\left(g_{1} H_{1} g_{1}^{-1} \cap g_{2} H_{2} g_{2}^{-1}\right)$. By minimality assumptions, $H_{1}$ and $H_{2}$ are conjugate.

TheOREM 1.14 (BOREL Density TheOREM [4]). If $G$ is a connected semisimple real algebraic group with no compact factors, and $S$ an ergodic $G$-space with finite invariant measure, then $S$ is Zariski dense in $G$.

As an example of an ergodic action of such a group, we point out the following example. (One can show there are uncountably many inequivalent actions of such groups [with finite invariant measure].) 
EXAMPLE 1.15. Let $\operatorname{SL}(n, \mathbf{Z})$ act on $\mathbf{R}^{n} / \mathbf{Z}^{n}$ by automorphisms. This is ergodic. The induced $\operatorname{SL}(n, \mathbf{R})$ action will be properly ergodic, essentially free (i.e. almost all stabilizers trivial), and have finite invariant measure.

2. Ergodicity theorems. A natural class of actions that arises in a variety of situations are actions on homogeneous spaces. Thus, if $H_{1}, H_{2} \subset G$ are subgroups with $\mathrm{H}_{2}$ closed, $H_{1}$ acts on $G / H_{2}$ and the question arises as to when this is ergodic. This is a special case of the following question. Suppose $S$ is an ergodic $G$-space and $H \subset G$ is a subgroup. When will the restriction to $H$ still be ergodic? In the special case in which $S$ has a finite invariant measure, results about unitary representations can be directly applied. Namely, let $\left(U_{g} f\right)(s)=$ $f(s g)$, where $f \in L^{2}(S)$. This defines a unitary representation of $G$ on $L^{2}(S)$ and $G$ is ergodic on $S$ (assuming finite invariant measure) if and only if there are no nonzero invariant vectors in $L^{2}(S) \ominus C$. Thus, to settle the question about ergodicity of restrictions in this case, we have a representation $U_{g}$ of $G$ with no invariant vectors and we ask whether or not $U \mid H$ has invariant vectors. Let us consider some of the classical examples, when $G$ is transitive on $S$.

EXAmple 2.1. Suppose $G$ is compact, $S=G$. Then $H \subset G$ is ergodic on $S$ if and only if $H$ is dense. This includes Example 1.2.

Now let $N$ be a simply connected nilpotent Lie group, $\Gamma \subset N$ a lattice (i.e., $\Gamma$ is discrete and $N / \mathrm{T}$ has finite invariant measure). For example,

$$
N=\left\{\left(\begin{array}{ccc}
1 & x & z \\
0 & 1 & y \\
0 & 0 & 1
\end{array}\right) \mid x, y, z \in \mathbf{R}\right\}
$$

and $\Gamma=N_{Z}$, the subgroup with $x, y, z \in Z$. Then $[N, N]=\{A \in N \mid x=y$ $=0\}$, and $N /[N, N] \Gamma$ is a torus. The map $N / \Gamma \rightarrow N /[N, N] \Gamma$ exhibits the 3-manifold $N / \Gamma$ as a circle bundle over the torus. In general $N / \Gamma \rightarrow$ $N /[N, N] \Gamma$ will be a bundle over the torus with fiber $[N, N] /[N, N] \cap \Gamma$.

Theorem 2.2 (L. Green [1]). $H \subset N$ is ergodic on $N / \Gamma$ if and only if it is ergodic on $N /[N, N] \Gamma$.

As the latter is a torus, ergodicity can be determined as in Example 2.1. The proof of this depends on writing down the representation of $N$ which appear in $L^{2}(N / \Gamma)$ and examining them with respect to restriction to subgroups. See [1] for details.

Results for 1-parameter subgroups acting on compact homogeneous spaces of solvable Lie groups have been obtained by Auslander [2] and Brezin and Moore [7].

If $G=\operatorname{SL}(2, \mathbf{R}), \Gamma \subset G$ is a lattice in $G$ and $H \subset G$ is the group of positive diagonal matrices, then $G / \Gamma$ is in a natural way the unit tangent bundle of the finite volume negatively curved manifold $D / \Gamma$ where $D=\operatorname{SO}(2, \mathbf{R}) \backslash G$ is the Poincare disk, and $H$ is the geodesic flow. Thus, a classical result of Hedlund [20] and Hopf [21] says that $H$ is ergodic on $G / \Gamma$. C. C. Moore generalized this to allow $G$ to be a very general semisimple Lie group, and $H$ to be an aribtrary subgroup. 
TheOREM 2.3 (C. C. MoORE [32]). Let $G=\prod G_{i}$ where $G_{i}$ is a noncompact connected simple Lie group with finite center and let $\Gamma \subset G$ be an irreducible latice. Then $H \subset G$ is ergodic on $G / \Gamma$ if and only if $\bar{H}$ is not compact.

This theorem was proved by showing the following general result about arbitrary representations (not necessarily one appearing in $L^{2}(G / \Gamma)$ ). Let $G$ be a noncompact connected simple Lie group with finite center and $\pi$ a unitary representation of $G$ with no nonzero invariant vectors. Then for any vector $x \neq 0,\{g \in G \mid \pi(g) x=x\}$ is compact. This result easily implies the theorem. A stronger result about such representations that we will need has subsequently come to light.

THEOREM 2.4. If $\pi$ is any unitary representation of a connected noncompact simple Lie group with finite center, then the matrix coefficients $f(g)=$ $\langle\pi(g) x \mid y\rangle \rightarrow 0$ as $g \rightarrow \infty$, assuming there are no $\pi(G)$-invariant vectors.

A nice proof of this appears in a paper of Howe and Moore [22] although the basic idea is present in the work of Sherman [43] (see also [49]). The idea of the proof is to let $G=K A K$ be a Cartan decomposition. Since $K$ is compact, it suffices to see $f(a) \rightarrow 0$ as $a \rightarrow \infty$. Consider the example $G=\operatorname{SL}(2, \mathbf{R})$, so that $A$ is the positive diagonals. Let $P$ be the upper triangular $2 \times 2$ matrices in $G$ with positive diagonal entries. The representation theory of $P$ is well known. There are 1-dimensional representations which factor through $[P, P]$ and 2 infinite dimensional representations induced from $[P, P]$. For the latter, it is clear that the restriction of a representation to $A$ is just the regular representation of $A$ for which it is clear that matrix coefficients vanish at $\infty$. Thus it suffices to see that $\pi \mid P$ has a spectral decomposition which assigns measure 0 to the 1-dimensional representations. But if it assigned positive measure, $[P, P]$ would have to leave a vector fixed, say $v$. Then $\varphi(g)=\langle\pi(g) v \mid v\rangle$ would be bi-invariant under $[P, P]=N . G / N$ can be identified with $\mathbf{R}^{2}-\{0\}$, and the $N$ orbits on $G / N$ are the horizontal lines except for the $x$-axis, and single points on the $x$-axis. A continuous function on $G / N$ constant on the orbits must clearly be constant on the $x$-axis as well. This translates into $\varphi(g)=1$ for all $g \in P$, and since $\pi$ is unitary, $v$ is $P$-invariant. Thus $\varphi$ is bi-invariant under $P$, and since $P$ has a dense orbit on $G / P, \varphi(g)=1$ for all $g \in G$, showing that $v$ is $G$-invariant.

We thus have good information about some basic examples for the question of ergodicity of actions on homogeneous spaces of finite invariant measure. For the general homogeneous space we make use of the following observation.

Proposition 2.5 [49]. If $S$ is an ergodic G-space (general quasi-invariant measure) and $H \subset G$ is a closed subgroup, then $H$ is ergodic on $S$ if and only if $G$ acts ergodically on the product $G / H \times S$.

To see this, suppose $A \subset G / H \times S$ is $G$-invariant. For each $x \in G / H$, let $A_{x}=\{s \in S \mid(x, s) \in A\}$. By quasi-invariance one easily sees that $A$ and all $A_{x}$ are simultaneously either null, of null complement, or neither. $A_{[e]}$ is an $H$-invariant set, and clearly any $H$-invariant set $B \subset S$ is of the form $B=A_{[e]}$ for some $G$-invariant $A$. 
Corollary 2.6 [32]. If $\Gamma, H \subset G$ are closed subgroups, then $H$ is ergodic on $G / \Gamma$ if and only if $\Gamma$ is ergodic on $G / H$.

This enables us to use information about ergodicity of restrictions on spaces for which there is a finite invariant measure to obtain results in the case no such measure exists.

Corollary 2.7 (Moore). $G=\Pi G_{i}, \Gamma$ as in Theorem 2.3. If $S$ is a transitive $G$-space, then $\Gamma$ is ergodic on $S$ if and only if the stabilizers in $G$ of points in $S$ are not compact.

EXAmple 2.8 (MoORE). $\operatorname{SL}(n, Z)$ is ergodic on $\mathbf{R}^{n}, n \geqslant 2$. This follows since $\operatorname{SL}(n, \mathbf{R})$ is essentially transitive on $\mathbf{R}^{n}$ and the stabilizers in the orbit of full measure are not compact.

Example 2.9. Consider the action of $\operatorname{SL}(2, \mathbf{R})$ on the Poincare disk $\operatorname{SL}(2, \mathbf{R}) / \operatorname{SO}(2, \mathbf{R})$. This action extends to the boundary circle, and the boundary can be identified with $\operatorname{SL}(2, \mathbf{R}) / P$, where $P$ is the upper triangular matrices in $G$. If $\Gamma \subset \operatorname{SL}(2, \mathbf{R})$ is a torsion free lattice, then $\Gamma$ acts in a properly discontinuous fashion on the disk, and the quotient space $D / \Gamma$ is a Riemann surface of finite volume. On the other hand, since $P$ is not compact, the action of $\Gamma$ on the boundary will be properly ergodic. More generally, if $G$ is any semisimple Lie group and $P \subset G$ is a minimal parabolic subgroup, then $G / P$ is the unique compact $G$-orbit in the boundary of a natural compactifiation of the symmetric space $X=G / K, K \subset G$ maximal compact. Here again, $\Gamma$ is ergodic on $G / P$. Thus these ergodic actions of $\Gamma$ on homogeneous spaces of $G$ arise very naturally in a geometric setting, and the study of these ergodic actions is extremely useful in understanding $\Gamma$.

Since this is such an important example, let us point out that for $G / P$ compact (e.g. $P$ a parabolic) that ergodicity of $\Gamma$ on $G / P$ can be demonstrated in a much less sophisticated fashion. Namely if there is a $P$-invariant vector in $L^{2}(G / \Gamma) \ominus \mathbf{C}$ then there is a compact $G$-orbit in the Hilbert space. As is well known, this implies that there exist finite dimensional subrepresentations, which for $G$, it is also well known, must be the identity. This is impossible.

Corollary 2.7 deals with the restriction of transitive $G$-actions to $\Gamma$. We now deal with the properly ergodic case.

THEOREM 2.10 [49]. If $G=\Pi G_{i}, G_{i}$ connected noncompact simple Lie groups with finite center, $\Gamma \subset G$ an irreducible lattice and $S$ is a properly ergodic $G$-space, then $\Gamma$ is ergodic on $S$.

Proof. Suppose not. Let $A \subset S \times G / \Gamma$ be invariant. For each $s$, let $f_{s} \in L^{2}(G / \Gamma) \ominus \mathbf{C}$ be the image under orthogonal projection of the characteristic function of $A_{s}=\{x \in G / \Gamma \mid(s, x) \in A\}$. We can suppose $f_{s} \neq 0$ on a set of positive measure. Invariance of $A$ is easily seen to imply that if we let $B \subset L^{2}(G / \Gamma) \ominus \mathbf{C}$ be the unit ball and let $G$ act on the right in $G$ via the unitary representation of $G$ on $L^{2}(G / \Gamma)$, then $\Phi: S \rightarrow B, \Phi(s)=f_{s}$ is a $G$-map. Then $\Phi_{*}(\mu)$ is a quasi-invariant ergodic measure on $B$.

But by vanishing of the matrix coefficients (Theorem 2.4), for $w \in B$, $w \cdot g \rightarrow 0$ weakly as $g \rightarrow \infty$. This implies $G$-orbits in $B$ are locally closed, i.e. 
the action is smooth. It follows that $\Phi_{*}(\mu)$ is supported on an orbit, so we can suppose $\Phi: S \rightarrow G / G_{0}$ where $G_{0}$ is the stabilizer of a point in this orbit. This implies $S=\operatorname{ind}_{G_{0}}^{G}\left(S_{0}\right)$ where $S_{0}$ is an ergodic $G_{0}$ space. But $G_{0}$ is compact, so $G_{0}$ is transitive on $S_{0}$. This implies $G$ is transitive on $S$, which contradicts our hypotheses.

Similar results can be proven for other groups for which there is a vanishing theorem for matrix coefficients.

THEOREM 2.11 [50]. Let $G$ be an exponential solvable Lie group and $S$ an ergodic $G$-space. Suppose $[G, G]$ is ergodic on $S$. Then $\Gamma$ is also ergodic on $S$ for every cocompact $\Gamma \subset G$.

The proof uses the result of Howe and Moore [22] that for such a group, the matrix coefficients $\langle\pi(g) v \mid w\rangle \rightarrow 0$ as $g \rightarrow \infty$ in $G / P_{\pi}$ where $P_{\pi}=\{g \mid \pi(g)$ is scalar $\}$. Here $\pi$ is assumed irreducible.

3. Cocycles. If $X$ is a $G$-space and $Y$ a Borel space, let $F(X, Y)$ be the space of measurable functions $X \rightarrow Y$, two functions being identified if they agree off a null set. $G$ acts on $X \times Y$ by $(x, y) \cdot g=(x g, y)$ and on $F(X, Y)$ by $(g \cdot f)(x)=f(x g)$. If $Y$ is also an $H$-space for some group $H$, we can define "twisted" actions. Namely, $(x, y) \cdot g=(x g, y \cdot \alpha(x, g))$ where $\alpha(x, g) \in H$, and for $f \in F(X, Y)$ (where for convenience we usually take $H$ to be acting on the left $),(g \cdot f)(x)=\alpha(x, g) f(x g)$. For these to define actions, we need the following compatibility condition: $\alpha(x, g h)=\alpha(x, g) \alpha(x g, h)$. Such a Borel function $\alpha: X \times G \rightarrow H$ will be called a cocycle. (The question as to whether this holds everywhere or almost everywhere is an important technical point which we will not discuss. See [41].) When endowed with this action we shall denote $X \times Y$ by $X \times{ }_{\alpha} Y$. If $Y=\mathscr{H}$ a Hilbert space, $H=U(\mathcal{H})$, the unitary group of the Hilbert space, and the measure on $X$ is invariant, then the $\alpha$-twisted action on $F(X, Y)$ restricts to $L^{2}(X ; \mathcal{H})$ to yield a unitary representation $U^{\alpha}$. If $\alpha, \beta: X \times G \rightarrow H$ are cocycles there is a certain relation which immediately implies equivalence of the actions or representations. Namely if we have a Borel map $\varphi: X \rightarrow H$ such that $\alpha(x, g)=\varphi(x) \beta(x, g) \varphi(x g)^{-1}$, this will be the case. We then call $\alpha$ and $\beta$ equivalent, or cohomologous, and write $\alpha \sim \beta$.

To get some further feeling for this notion, consider the case $X=G / G_{0}$. If $\alpha: G / G_{0} \times G \rightarrow H$ is a cocycle, then $\alpha \mid[e] \times G_{0}$ defines a homomorphism $G_{0} \rightarrow H$. Equivalent cocycles yield conjugate homomorphisms. Furthermore, every homomorphism $G_{0} \rightarrow H$ arises from a cocycle $\alpha$ in this way. Namely, let $\gamma: G / G_{0} \rightarrow G$ be a Borel section. Then for $(x, g) \in G / G_{0} \times G, \gamma(x g)$ and $\gamma(x) \cdot g$ are equal when projected to $G / G_{0}$. Thus $\gamma(x) g \gamma(x g)^{-1} \in G_{0}$. We can suppose $\gamma([e])=e$, and then $(x, g) \rightarrow \gamma(x) g \gamma(x g)^{-1}$ is a cocycle $G / G_{0} \times G \rightarrow$ $G_{0}$ which when restricted to $[e] \times G_{0}$ yields the identity $G_{0} \rightarrow G_{0}$. Thus if $\pi$ : $G_{0} \rightarrow H$ is a homomorphism, $\alpha(x, g)=\pi\left(\gamma(x) g \gamma(x g)^{-1}\right)$ is the required cocycle. Thus we have

THEOREM 3.1. $\alpha \rightarrow \alpha \mid[e] \times G_{0}$ defines a bijection between equivalence classes of cocycles $G / G_{0} \times G \rightarrow H$ and conjugacy classes of homomorphisms $G_{0} \rightarrow H$. 
We remark that if $H=U(\mathcal{H})$, and $\pi: G_{0} \rightarrow H$ is a unitary representation, we have an associated cocycle $\alpha: G / G_{0} \times G \rightarrow H$, and then an associated representation $U^{\alpha}$ of $G$ on $L^{2}\left(G / G_{0} ; \mathcal{G}\right)$. Of course $U^{\alpha}=\operatorname{ind}_{G_{0}}^{G}(\pi)$. See [45] for this approach to induced representations.

We now consider some other examples.

EXAMPLE 3.2. (a) If $h: G \rightarrow H$ is a homomorphism, $X$ a $G$-space, then $\alpha(x, g)=h(g)$ is a cocycle. If $X=G / G_{0}$, this corresponds to a homomorphism $G_{0} \rightarrow H$, which is simply $h \mid G_{0}$. Thus in general we shall sometimes call $\alpha$ the restriction of $h$ to $X \times G$ and write $\alpha=h \mid X \times G$.

(b) Suppose $X$ is an ergodic $G$-space with quasi-invariant measure $\mu$. Let $r_{\mu}(x, g)=d \mu(x g) / d \mu(x)$, the Radon-Nikodym derivative. The chain rule implies $r_{\mu}: X \times G \rightarrow R^{+}$is a cocycle, called the Radon-Nikodym cocycle. If $\mu \sim \nu$, so $d \mu=f d \nu, f>0$, then

$$
d \mu(x g) / d \mu(x)=f(x)^{-1}(d \nu(x g) / d \nu(x)) f(x g),
$$

i.e., $r_{\mu} \sim r_{\nu}$. Therefore the cohomology class we obtain does not depend upon the measure, only the measure class. In particular, there is an equivalent $\sigma$-finite invariant measure if and only if the cocycle is trivial (i.e. equivalent to the identity $\alpha(x, g)=1)$.

(c) Suppose $X$ is a $G$-space, $X^{\prime}$ a free $G^{\prime}$-space, and that the actions are orbit equivalent, with $\theta: X \rightarrow X^{\prime}$ the orbit equivalence. Then for $(x, g) \in X \times G$, $\theta(x)$ and $\theta(x g)$ are in the same $G^{\prime}$-orbit, say $\theta(x) \alpha(x, g)=\theta(x g)$ for $\alpha(x, g)$ $\in G^{\prime}$. Then $\alpha: X \times G \rightarrow G^{\prime}$ is a cocycle. If $G=G^{\prime}$, we have the following.

Proposition 3.3 [55]. If $\alpha$ is equivalent to the restriction of an automorphism $A \in \operatorname{Aut}(G)$ to $X \times G$, then $X$ and $X^{\prime}$ are automorphically conjugate. If this automorphism is inner, then $X$ and $X^{\prime}$ are conjugate.

Proof. If $\alpha(x, g)=\lambda(x) A(g) \lambda(x g)^{-1}$, then $\theta_{1}(x)=\theta(x) \lambda(x)$ satisfies $\theta_{1}(x g)=\theta_{1}(x) A(g)$, so we have automorphic conjugacy. If $A(g)=h g h^{-1}$, let $\theta_{2}(x)=\theta_{1}(x) h$. This is then a $G$-map.

There are many other naturally arising situations in which cocycles appear, but we shall not have time to discuss them here. Instead, we turn to an important invariant attached to a cocycle, namely the Mackey range. Let $\alpha$ : $S \times G \rightarrow H$ where $H$ is also locally compact. Form the twisted $G$-action $S \times{ }_{\alpha} H$ where we view $H$ as acting on itself by right translations. $H$ also acts on $S \times_{\alpha} H$ by $(s, h) \cdot h_{0}=\left(s, h_{0}^{-1} h\right)$, and this $H$ action commutes with the $G$-action. Note that if $i: G \rightarrow H$ is an embedding of $G$ into a larger group and $\alpha=i \mid S \times G$, this is exactly the situation in the inducing procedure. As in the latter, we obtain an action of $H$ on the space of $G$-orbits. But this space may not be a decent measure space, so instead, we let $X$ be the space of $G$-ergodic components of the action of $G$ of $S \times{ }_{\alpha} H$. Then $H$ will act on $X$ as well, and this will be an ergodic $H$-action.

Definition 3.4. If $\alpha: S \times G \rightarrow H$ is a cocycle, the associated $H$-space $X$ will be called the Mackey range of $\alpha$. This is a cohomology invariant of $\alpha$.

EXAMPLE 3.5. (a) If $i$ : $G \rightarrow H$ is an embedding of $G$ as a closed subgroup of $H$, and $\alpha(s, g)=i(g)$, i.e. $\alpha=i \mid S \times G$, then the Mackey range of $\alpha$ is $\operatorname{ind}_{G}^{H}(S)$. 
(b) If $\theta: X \rightarrow X^{\prime}$ is an orbit equivalence, $\alpha: X \times G \rightarrow G^{\prime}$ the associated cocycle, then the Mackey range is the $G^{\prime}$-space $X^{\prime}$.

(c) If $S=G / G_{0}$ and $\alpha$ corresponds to a homomorphism $\pi$ : $G_{0} \rightarrow H$, then the Mackey range of $\alpha: G / G_{0} \times G \rightarrow H$ is the $H$-space $H / \overline{\pi\left(G_{0}\right)}$.

Finally, the following relates the Mackey range to the cohomology class of $\alpha$.

Proposition 3.6. If $\alpha$ : $S \times G \rightarrow H$, the following are equivalent.

(i) $\alpha \sim \beta$ where $\beta(S \times G) \subset H_{0}, H_{0} \subset H$ a closed subgroup.

(ii) $\mathrm{H} / \mathrm{H}_{0}$ is a factor of the Mackey range.

(iii) $X=\operatorname{ind}_{H_{0}}^{H}\left(S_{0}\right)$ for some $S_{0}$, where $X$ is the Mackey range.

For a proof, see $[47,52]$.

4. Generalized discrete spectrum. Suppose $(S, \mu)$ is an ergodic space with $\mu$ finite and invariant. In this lecture we try to see what the algebraic structure of the representation $\pi$ of $G$ on $L^{2}(S)$ says about the geometric structure of the action.

Definition 4.1. We say that the action has discrete spectrum if $\pi$ is the direct sum of finite dimensional irreducible subrepresentations.

EXAMPLE 4.2. Let $K$ be a compact group, $H$ a closed subgroup, and $\varphi$ : $G \rightarrow K$ a homomorphism with $\varphi(G)$ dense in $K$. Let $G$ act on $K / H$ by $[k] \cdot g=[k \varphi(g)]$. Then this action has discrete spectrum.

Theorem 4.3 (VON Neumann-Halmos-Mackey). These are all the examples. That is, if $S$ is a $G$-space with discrete spectrum, then there exists a compact group $K$, a closed subgroup $H$, and a homomorphism $\varphi: G \rightarrow K$ with dense range such that $S$ and $K / H$ are conjugate $G$-spaces.

This was originally proved by von Neumann and Halmos for $G=Z$ or $\mathbf{R}$, and by Mackey [26] for general $G$. We sketch Mackey's proof.

Let $L^{2}(S)=\Sigma^{\oplus} W_{i}$ where $W_{i}$ are $\pi(G)$-invariant and finite dimensional. Let $B=\Pi U\left(W_{i}\right)$, the product of the associated unitary groups, which is a compact subgroup of $U\left(L^{2}(S)\right)$. Further, $\pi: G \rightarrow B$. Let $K=\overline{\pi(G)}$, so that $K$ is also compact. Let $M$ be the abelian von Neumann algebra on $L^{2}(S)$ consisting of multiplication by elements of $L^{\infty}(S)$. Then clearly $\pi(g) M \pi(g)^{-1}=M$, and by passing to the strong limit, we obtain $T M T^{-1}=M$ for all $T \in K$. From this one can deduce that each operator $T$ in $K$ is induced by a point transformation of $S$, and thus the $G$-action on $S$ extends to an action of $K$. (There is some delicate measure theory we are ignoring here.) Since the $G$-action is already ergodic, so is the $K$-action. Since $K$ is compact, $K$ must act transitively, so we can identify $S \cong K / H$.

Theorem 4.3 can be generalized to extensions. Namely, suppose $X \rightarrow Y$ is an extension of ergodic $G$-spaces with finite invariant measure. The Hilbert space $L^{2}(X)$ not only has a natural representation of $G$ on it, but $L^{2}(X)$ is also an $L^{\infty}(Y)$-module in a natural way. (Namely, lift a function on $Y$ to a function on $X$ and multiply.) Alternatively, we can express this by saying that there is a natural system of imprimitivity for $\pi$ on $L^{2}(X)$ based on $Y$. 
Definition 4.4 [47]. We say that $X$ has relatively discrete spectrum over $Y$ if $L^{2}(X)=\Sigma^{\oplus} \bar{W}_{i}$ where $W_{i}$ are $G$-invariant subspaces that are finitely generated as $L^{\infty}(Y)$-modules.

EXAMPLE 4.5. Suppose $Y$ is an ergodic $G$-space with finite invariant measure, $\alpha: Y \times G \rightarrow K$ is a cocycle where $K$ is compact, and $H \subset K$ is a closed subgroup. Then $X=Y \times{ }_{\alpha} K / H$ is an extension of $Y$ with relatively discrete spectrum. To see this, observe that $L^{2}(X)=L^{2}\left((Y) ; L^{2}(K / H)\right)$. Write $L^{2}(K / H)=\Sigma^{\oplus} Z_{i}$ where $Z_{i}$ are finite dimensional and $K$-invariant. We then have $L^{2}(X)=\Sigma^{\oplus} L^{2}\left(Y ; Z_{i}\right)$ and $L^{2}\left(Y ; Z_{i}\right)$ will be $G$-invariant since $G$ acts from fiber to fiber in $X$ by an element of $K$, and $Z_{i}$ is $K$-invariant. Clearly $L^{2}\left(Y ; Z_{i}\right)=\overline{L^{\infty}\left(Y, Z_{i}\right)}$ and the latter is finitely generated over $L^{\infty}(Y)$.

THEOREM 4.6 [47]. These are all the examples. That is, if $X \rightarrow Y$ is an ergodic extension with relatively discrete spectrum, then there exists a compact group $K$, a closed subgroup $H \subset K$, and a cocycle $\alpha: Y \times G \rightarrow K$, such that as extensions of $Y, X \cong Y \times{ }_{\alpha} K / H$.

Thus Theorem 4.6 tells us how to recognize extensions of the form $Y \times{ }_{\alpha} K / H$ from information about the unitary representation of the extension. There is now a larger class of actions whose "structure" we know.

Definition 4.7 [48]. We say that $X$ has generalized discrete spectrum if $X$ can be built from a point via the operations of taking extensions with relatively discrete spectrum and inverse limits. More precisely, there is a countable ordinal $\alpha$ and for each $\sigma \leqslant \alpha$ a factor $X_{\sigma}$ of $X$ such that

(i) $X_{0}=$ point,

(ii) $X_{\sigma+1} \rightarrow X_{\sigma}$ is an extension with relatively discrete spectrum, for $\sigma<\alpha$,

(iii) if $\sigma$ is a limit ordinal, $X_{\sigma}=\lim _{\leftarrow}\left\{X_{\beta}, \beta<\sigma\right\}$,

(iv) $X_{\alpha}=X$.

In light of Theorem 4.6, we have an exact picture of the structure of such actions. We would now like to see which actions arise in this fashion.

DEFINITION 4.8. If $G$ acts continuously on a compact metric space $X, G$ is called distal on $X$ if $x, y \in X, x \neq y$, implies inf $\operatorname{s\in G}_{g \in G} d(x g, y g)>0$.

Clearly any isometric action is distal. However, not every distal action admits an invariant metric. For example, if $N$ is a nilpotent Lie group and $\Gamma \subset N$ is a lattice, then the action of $N$ on $N / \Gamma$ is distal. This was first shown in [1].

Definition 4.9 (PARRY [38]). If $(S, \mu)$ is an ergodic $G$-space, call the action measure distal if there is a decreasing sequence of sets of positive measure $\left\{A_{i}\right\}$ with $\mu\left(A_{i}\right) \rightarrow 0$, such that if $x, y \in S, x g_{i}, y g_{i} \in A_{i}$ for some sequence $g_{i} \in G$, then $x=y$. (We have ignored some measure theoretic issues in this definition, which arise if $G$ is not discrete. See [48] for a more careful formulation.)

Any distal action with an invariant measure that is positive on open sets is clearly measure distal.

TheOREM 4.10 [48]. A finite measure preserving ergodic action (on a nonatomic measure space) is measure distal if and only if it has generalized discrete spectrum. 
This is an analogue for measure theoretic actions of the Furstenberg structure theorem for minimal (i.e. every orbit dense) distal actions on compact metric spaces [16].

Another situation in which actions with generalized discrete spectrum arise is the following.

TheOREM 4.11. Let $N$ be a nilpotent group. Suppose $S$ is an ergodic $N$-space for which $L^{2}(S)$ is a direct sum of irreducible representations (not necessarily finite dimensional). Then $S$ has generalized discrete spectrum (and the ordinal in Definition 4.7 can be taken to be finite).

This theorem is false for solvable groups. Let us give an example of such a properly ergodic $N$-space. Let $N$ be the Heisenberg group, $N_{Z}=\Gamma$ the integer points, so that $\Gamma$ is a lattice. There is an injective homomorphism $\Gamma \rightarrow K$ where $K$ is compact. For example, let $K=\prod N_{Z / p Z}$, where the product is taken over all primes. Then $K$ is a $\Gamma$-space with discrete spectrum. Let $X=\operatorname{ind}_{\Gamma}^{N}(K)$. Since a finite dimensional representation of $\Gamma$ induced to $N$ decomposes into a direct sum of irreducibles, and $\pi$ on $L^{2}(X)$ can be expressed as $\pi=\operatorname{ind}_{\Gamma}^{N}(\sigma)$ where $\sigma$ is the representation of $\Gamma$ on $L^{2}(K)$, it follows that $L^{2}(X)$ is a direct sum of irreducibles.

It is natural to ask which groups have actions of the sort we have been discussing in a nontrivial way, say an effective or essentially free action. A group will have an effective or free action with discrete spectrum if and only if there are enough finite dimensional unitary representations to separate points. In the connected case, such groups are identified by a classical theorem of Freudenthal.

Theorem 4.12 (Freduenthal [14]). A connected group has a free (or effective) action with discrete spectrum if and only if it is isomorphic to $\mathbf{R}^{n} \times K$ where $K$ is compact.

To describe the analogous result for generalized discrete spectrum, we recall that a connected group is said to be of polynomial growth if for any compact neighborhood of the identity, $W$, the Haar measure $m\left(W^{n}\right)$ grows no faster than a polynomial in $n$. (If this is true for one compact neighborhood, it is true for all such neighborhoods.) For Lie groups, this condition is equivalent to the group being of type $(\mathrm{R})[19,23]$. We recall that this means that every eigenvalue of $\operatorname{Ad}(g)$ lies on the unit circle for all $g \in G$. For example, nilpotent groups and Euclidean motion groups are type $(\mathrm{R})$, while semisimple groups and the $a x+b$ group are not. The following is joint work with $\mathrm{C}$. C. Moore.

THEOREM 4.13 [34]. A connected group has a free (or effective) ergodic action with generalized discrete spectrum if and only if it is of polynomial growth.

Proof. We indicate the proof for the $a x+b$ group. The general proof is based on this argument and some structure theory for Lie groups, particularly that of solvable Lie groups.

Let $G=A B$ be a semidirect product where $B=\mathbf{R}$ is normal and $A=\mathbf{R}^{+}$ acts on $B$ by multiplication. If $X$ is a $G$-space with generalized discrete 
spectrum, and $X_{1}$ is the factor of $X$ with discrete spectrum, then $B$ must act trivially on $X_{1}$ since all finite dimensional unitary representations of $G$ are one dimensional and thus factor through $B=[G, G]$. It therefore suffices to show the following: suppose $\varphi: X \rightarrow Y$ is an extension of $G$-spaces with relatively discrete spectrum and suppose $B$ acts trivially on $Y$; then $B$ acts trivially on $X$. To prove this assertion, let $L^{2}(X)=\Sigma^{\oplus} \vec{W}_{i}, W_{i}$ a finitely generated $L^{\infty}(Y)$ module which is $G$-invariant. Let $\mu, \nu$ be the given measures on $X$ and $Y$ respectively, and decompose $\mu$ with respect to $\nu$ over the fibers of $\varphi$. Thus, we write $\mu=\int \mu_{y} d \nu(y)$ where $\mu_{y}$ is supported on $\varphi^{-1}(y)$. This gives us a direct integral decomposition $L^{2}(X)=\int_{Y}^{\oplus} L^{2}\left(\varphi^{-1}(y), \mu_{y}\right) d \nu$. For each $y, g$ we have $\alpha(y, g): L^{2}\left(\varphi^{-1}(y g), \mu_{y g}\right) \rightarrow L^{2}\left(\varphi^{-1}(y), \mu_{y}\right)$ given by $[\alpha(y, g) f](z)=f(z g)$ for $z \in \varphi^{-1}(y)$. Fix $i$. Saying that $W_{i}$ is finitely generated over $L^{\infty}(Y)$ and $G$-invariant means that there is $V_{y} \subset L^{2}\left(\varphi^{-1}(y)\right)$, a finite dimensional subspace, such that $W_{i}=\int^{\oplus} V_{y} d \nu(y)$ and $\alpha(y, g) V_{y g}=V_{y}$. For $g \in B, y g=y$, so $\alpha \mid\{y\} \times B$ is a unitary representation of $B$ on $V_{y}$. Say $\operatorname{dim} V_{y}=n$. Then for each $y$, we have $n$ elements in $\hat{B}=$ character group of $B$. Furthermore, $G$ acts on $\hat{B}$ and one can check that the cocycle identity for $\alpha$ implies

$(\alpha \mid\{y\} \times B) \cdot g \cong \alpha \mid\{y g\} \times B$, where $\cong$ means unitary equivalence.

Let $\hat{B}^{n} / S_{n}$ ( $S_{n}$ is the symmetric group on $n$ letters) be the set of unordered $n$-tuples of elements of $\hat{B}$. We have a map $\Phi: Y \rightarrow \hat{B}^{n} / S_{n}$, and $(*)$ implies that $\Phi$ is a $G$-map. The action of $G$ on $\hat{B} \cong \mathbf{R}$ has three orbits, namely the origin and the 2 half lines. From this it is easy to see that every $G$-orbit in $\hat{B}^{n}$ (and hence in $\hat{B}^{n} / S_{n}$ ) is locally closed, and that the only compact $G$-orbit is the identity (i.e. the origin). But $\Phi_{*}(\mu)$ is a finite invariant ergodic measure on $\hat{B}^{n} / S_{n}$. By smoothness, this must be supported on an orbit and by finiteness and invariance, this must clearly be the zero orbit. Thus, $\alpha \mid\{y\} \times B$ is the identity for all $y$, so $B$ acts trivially on each $W_{i}$. Therefore, $B$ is trivial on $L^{2}(X)$ and hence on $X$ as well.

Finally, we remark that the notion of generalized discrete spectrum yields a type of structure theorem for general actions with finite invariant measure that is sometimes useful. If $S$ is an ergodic $G$-space, it is not always true that $S \times S$ is also ergodic, where $G$ acts by $(s, t) g=(s g, t g)$. If this additional ergodicity property holds, the action is called weakly mixing. More generally, if $X \rightarrow Y$ is an ergodic extension of $Y$, the fibered product $X \times_{Y} X$ has a natural $G$-invariant measure on it [47], but this action no longer need be ergodic. Once again, if this extra ergodicity holds, the extension $X$ is called relatively weak mixing over $Y$. Given any ergodic $G$-space $X$, there is a unique maximal factor $Z$ of $X$ such that $Z$ has generalized discrete spectrum and $X$ is relatively weakly mixing over $Z$. Thus we break $X$ up into a factor whose structure we know explicitly, and an extension with extra ergodicity properties. Of course, simply by knowing that an action or extension is weak mixing does not say very much about its detailed structure, so for most questions, this is not a satisfactory structure theorem aside from the factor $Z$. Nevertheless, weak mixing does clearly have some information, and thus one can hope to find this decomposition useful in some circumstances. An example of this appears in recent work 
of Furstenberg. Szemeredi recently succeeded in proving a conjecture of Erdös which asserts that every set of positive integers of positive upper density contains arithmetic progressions of arbitrary (finite) length. In [17], Furstenberg gave another proof of Szemeredi's theorem, first by converting this to a statement about measure preserving integer actions, and then proving the latter by proving it first for actions with generalized discrete spectrum, and then showing the property is preserved by passing to relatively weakly mixing extensions.

5. Amenability. The notion of an amenable group can be described in a variety of ways. Here, we shall focus on the fixed point property.

Let $E$ be a separable Banach space, $E^{*}$ the dual, $E_{1}^{*}$ the unit ball in $E^{*}$, and Iso $(E)$ the group of isometric isomorphisms of $E$. Suppose $\pi: G \rightarrow \operatorname{Iso}(E)$ is a representation of $G$ on $E$, and that $A \subset E_{1}^{*}$ is a compact convex $G$-invariant set. (Here $G$ acts on $E^{*}$ via the adjoint representation, $\pi^{*}(g)=\left(\pi\left(g^{-1}\right)\right)^{*}$.)

Definition 5.1. $G$ is amenable if for all $\pi$ and $A$ as above, there is a fixed point for $G$ in $A$.

For example, if $G$ is amenable and $G$ acts continuously on a compact metric space $X$, then there is a $G$-invariant probability measure on $X$. We simply apply the definition to $E=C(X)$ where $A \subset C(X)^{*}$ is the set of probability measures. In fact a standard convexity argument shows that $G$ is amenable if and only if there is a $G$-invariant measure on every compact metric $G$-space.

Abelian groups are amenable by the Markov-Kakutani fixed point theorem, and compact groups are easily seen to be amenable. If $0 \rightarrow A \rightarrow B \rightarrow C \rightarrow 0$ is an exact sequence, then $B$ is amenable if and only if $A$ and $C$ are amenable. Thus, groups with a cocompact solvable normal subgroup are amenable. Every connected amenable group is of this form, but this is no longer true among all discrete groups [18].

We now wish to define the notion of an amenable ergodic action of a group, originally introduced in [51]. This will include all actions of amenable groups, as well as some actions of nonamenable groups. We begin by describing certain classes of $G$-invariant compact convex sets that arise from an ergodic $G$-space $S$.

So suppose $S$ is an ergodic $G$-space and $\alpha: S \times G \rightarrow \operatorname{Iso}(E)$ is a cocycle. We then have the adjoint cocycle $\alpha^{*}(s, g)=\left(\alpha(s, g)^{-1}\right)^{*}$, and the $\alpha^{*}$-twisted action on $L^{\infty}\left(S, E^{*}\right)$, given by $(g \cdot f)(s)=\alpha^{*}(s, g) f(s g)$, for $f \in L^{\infty}\left(S, E^{*}\right)$. We observe that $L^{\infty}\left(S, E^{*}\right)=\left(L^{1}(S, E)\right)^{*}$, so that $L^{\infty}\left(S, E^{*}\right)$ is a dual space. We want to describe certain $G$-invariant compact convex sets in the unit ball of this dual space. One natural possibility is to take $A \subset E_{1}^{*}$ compact, convex, and satisfying the condition $\alpha^{*}(s, g) A=A$. Then $F(S, A)$ (= measurable functions $S \rightarrow A$ ) will be a compact convex $G$-invariant set in $L^{\infty}\left(S, E^{*}\right)$. However, it is also possible to vary the set $A$ as we move from point to point in $S$. Thus, suppose $\left\{A_{s}\right\}$ is a collection of compact convex subsets $A_{s} \subset E_{1}^{*}$, which vary measurably in $s$, and satisfying the condition $\alpha^{*}(s, g) A_{s g}=A_{s}$. Then $F\left(S,\left\{A_{s}\right\}\right)=\left\{f \in L^{\infty}\left(S, E^{*}\right) \mid f(s) \in A_{s}\right\}$ is a compact convex $G$ invariant set. 
Definition 5.2 [51]. Call a set of the form $F\left(S,\left\{A_{s}\right\}\right)$ a compact convex set over $S$. An ergodic action of $G$ on $S$ is called amenable if every compact convex $G$-invariant set over $S$ has a fixed point.

Thus while amenability of $G$ demands a fixed point in every compact convex set, amenability of the action demands a fixed point only in compact convex sets over the action. We also remark that the condition that one has a fixed point simply means $\alpha^{*}(s, g) f(s g)=f(s)$ for $f: S \rightarrow E^{*}, f(s) \in A_{s}$. As an example of how one can use this condition, suppose $S$ is an amenable $G$-space and that $X$ is a compact metric $G$-space. Let $M(X)$ be the space of probability measures on $X$. We have a representation $\pi: G \rightarrow \operatorname{Iso}(C(X))$ and hence a cocycle $\alpha: S \times G \rightarrow \operatorname{Iso}(C(X))$ by restriction, i.e. $\alpha(s, g)=\pi(g) . M(X)$ will be a $G$-invariant compact convex set, and thus we can take $A_{s}=M(X)$ for all $s$. (So for this example, we did not have to vary the compact convex set in going from point to point.) Amenability of the action then implies that there is a function $f: S \rightarrow M(X)$ such that $\alpha^{*}(s, g) f(s g)=f(s)$, i.e., $\pi^{*}(g) f(s g)=f(s)$. Switching to a right action on $M(X)$, we obtain that $f(s g)=f(s) \cdot g$. Thus, we conclude that if $S$ is an amenable ergodic $G$-space, $X$ a compact metric $G$-space, then there is a measurable $G$-map $f: S \rightarrow M(X)$.

We now list some basic properties. Proofs can be found in [51, 52].

Proposition 5.3. (a) If $G$ is amenable, every ergodic $G$-space is amenable.

(b) If $S$ is an amenable ergodic $G$-space with finite invariant measure, then $G$ is amenable.

(c) If $S=G / H$, then $G / H$ is an amenable $G$-space if and only if $H$ is amenable.

(d) If $S$ is an amenable ergodic $G$-space, and $\Gamma \subset G$ is a closed subgroup, then the restriction of the action on $S$ to $\Gamma$ is amenable.

EXAMPLE 5.4. Let $\Gamma \subset \operatorname{SL}(2, \mathbf{R})$ be a lattice and consider the ergodic action of $\Gamma$ on the boundary circle of the Poincaré disk. This is just the action of $\Gamma$ on $\mathrm{SL}(2, \mathbf{R}) / P$ where $P$ is the upper triangular subgroup. Since $P$ is amenable, the $\Gamma$-action on the boundary circle is amenable by (c) and (d) of the above proposition. More generally, let $G$ be a semisimple Lie group, $\Gamma \subset G$ a lattice, and $P \subset G$ a minimal parabolic subgroup. Then $P$ is amenable and so $\Gamma$ acting on $G / P$ is amenable.

This example indicates how natural and important examples of actions of nonamenable groups are amenable. Assertion (c) of the above proposition shows that any group has amenable transitive actions. More generally, we have the following.

Proposition 5.5 [52]. If $H \subset G$ is a closed subgroup and $S$ is an ergodic $H$-space, then $S$ is an amenable $H$-space if and only if $\operatorname{ind}_{H}^{G}(S)$ is an amenable G-space.

This proposition raises the following question. Although one can have amenable actions of nonamenable groups, does every such action come in a simple way from an action of an amenable subgroup, namely just by inducing? In fact, the answer in general is no. One can show that if $\Gamma \subset \operatorname{SL}(2, C)$ is a 
lattice, then $\Gamma$ is amenable on $\mathbf{C P}^{1}$ (this is just Example 5.4) but that this action is not induced from an action of an amenable subgroup. This latter fact is not trivial, and a proof is given in [52]. On the other hand, we do have the following.

TheOrem $5.6[52,55]$. Let $G$ be a connected group. Then every amenable ergodic action of $G$ is induced from an action of an amenable subgroup.

Proof. We give the proof for $G$ semisimple. Let $S$ be an amenable $G$-space and $P \subset G$ be a minimal parabolic subgroup. Since $G / P$ is compact, by the remarks following Definition 5.2, there is a measurable $G$-map $f: S \rightarrow M(G / P)$, where the latter is the space of probability measures on $G / P$. The proof will now follow from two basic results about the action of $G$ on $M(G / P)$. The first is due to the author, the second to C. C. Moore [33].

THEOREM $5.7[52,55]$. Let $G$ be a connected semisimple Lie group and $P \subset G$ any parabolic subgroup. Then every element in $M(G / P)$ has a locally closed orbit under the G-action.

Theorem 5.8 (C. C. Moore [33]). Let $G$ be a connected semisimple Lie group with trivial center and $P \subset G$ a minimal parabolic subgroup. Let $\mu \in M(G / P)$ and $G_{\mu}$ the stabilizer of $\mu$ in $G$. Then $G_{\mu}$ is an amenable algebraic group. (By algebraic, we mean the intersection of $G$ with an algebraic subgroup of $\tilde{G}$, where the latter is an algebraic subgroup containing $G$ as a subgroup of finite index.)

To conclude the proof of Theorem 5.6 given these results, we simply observe that Theorem 5.7 asserts the smoothness of the $G$-action on $M(G / P)$, so if $m$ denotes the measure on $S$, then $f_{*}(m)$ is quasi-invariant and ergodic on $M(G / P)$ and hence supported on an orbit. Thus we can consider $f$ as $f$ : $S \rightarrow G / H$, where $H$ is the stabilizer of an orbit. But by Theorem 5.8, $H$ is amenable so the result follows from Proposition 1.11.

Without giving a proof of Theorems 5.7 and 5.8, let us at least try to give some indication of why they are true. To this end, we state the following lemma of Furstenberg [15].

Lemma 5.9 (Furstenberg). Suppose $g_{n} \in \mathrm{SL}(n, \mathbf{R}), g_{n} \rightarrow \infty$. Let $\mu \in$ $M\left(\mathbf{P}^{n-1}\right)$ and suppose $\mu \cdot g_{n} \rightarrow \nu$ for some $\nu \in M\left(\mathbf{P}^{n-1}\right)$. Then $\nu$ is supported on a union of two proper projective subspaces.

REMARK. (i) Since the set of measures supported on a union of two proper projective subspaces is closed, this shows that the orbit of any $\mu$ not so supported is locally closed. This is the beginning of an inductive procedure for proving Theorem 5.7 .

(ii) If $H \subset \operatorname{SL}(n, \mathbf{R})$ leaves a measure fixed, then $H$ is either compact or leaves the union of finitely many proper subspaces invariant. Both of these conditions are "algebraic". Proceeding inductively, one should obtain either compactness or further splitting. This should result in an algebraic object which is a compact extension of a solvable group. In this way, Lemma 5.9 suggests Theorem 5.8 as well. 
Proof of Lemma 5.9. Let $h_{n}=g_{n} /\left\|g_{n}\right\|$. Then $\left\|h_{n}\right\|=1, \operatorname{det}\left(h_{n}\right) \rightarrow 0$, so we can assume $h_{n} \rightarrow h, h \neq 0, \operatorname{det}(h)=0$. Let $V=\operatorname{range}(h), N=\operatorname{ker}(h),[V]$, $[N]$ the corresponding projective subspaces in $\mathbf{P}^{n-1}$. Write $\mu=\mu_{1}+\mu_{2}$ where $\operatorname{support}\left(\mu_{1}\right) \subset[N], \operatorname{support}\left(\mu_{2}\right) \subset \mathbf{P}^{n-1}-[N]$. If $x \in \mathbf{P}_{n-1}-[N]$, then as $n \rightarrow \infty, h_{n}(x) \rightarrow h(x) \in[V]$. Passing to a subsequence, we can write

$$
\nu=\lim \mu \cdot g_{n}=\lim \mu_{1} \cdot g_{n}+\lim \mu_{2} \cdot g_{n},
$$

and the previous sentence implies $\lim \mu_{2} \cdot g_{n}$ is supported on [V]. We also have $\lim \mu_{2} \cdot g_{n}$ supported on $[W]$ where $\lim [N] \cdot g_{n}=[W]$.

We now turn to the theory of orbit equivalence for amenable actions. We begin with an observation.

Proposition 5.10. For free ergodic actions amenability is an invariant of orbit equivalence.

The circle of ideas concerning orbit equivalence began in the late 1950s with the work of $\mathrm{H}$. Dye $[\mathbf{1 0}, \mathbf{1 1}]$ and for amenable actions has been brought to complete form very recently. We begin with the fundamental theorem of Dye.

TheOREM 5.11 (Dye [10]). All finite measure preserving (properly) ergodic Z-actions are orbit equivalent.

This was later extended to the $\sigma$-finite case by Krieger [25].

TheOrem 5.12 (Krieger). All $\sigma$-finite (but not finite) measure preserving Z-actions are orbit equivalent.

Krieger also extended the theorem to the case of quasi-invariant measure without equivalent invariant measure. He showed that a measurement of the extent to which the action fails to be measure preserving is a complete invariant of orbit equivalence. Namely, let $X$ be an ergodic $G$-space, and $r$ : $X \times G \rightarrow \mathbf{R}^{+}$be the Radon-Nikodym cocycle. Let $\Delta: G \rightarrow \mathbf{R}^{+}$be the modular function of $G$, and let $m: X \times G \rightarrow \mathbf{R}^{+}$be $m(x, g)=r(x, g) \Delta(g)^{-1}$. We call $m$ the modular cocycle. The Mackey range of this cocycle will be an ergodic $\mathbf{R}^{+}$-action, which we call the modular flow or the modular range. For unimodular groups, the modular flow will be translation of $\mathbf{R}^{+}$on $\mathbf{R}^{+}$itself if and only if there is an invariant measure for the action (for then the Radon-Nikodym cocycle is trivial (Proposition 3.6)).

Theorem 5.13 (KrIEGER). For Z-actions with quasi-invariant measure, and not possessing (an equivalent) finite invariant measure, the modular flow is a complete invariant of orbit equivalence.

A good account of Krieger's work is [44].

EXAMPLE 5.14. Let us see how to compute the modular flow in some examples. Let $\Gamma \subset \operatorname{SL}(2, \mathbf{R})$ be a lattice, $P$ the upper triangular subgroup. The Radon-Nikodym cocycle for the action of $G=\operatorname{SL}(2, \mathbf{R})$ on $G / P$ is the cocycle $\alpha: G / P \times G \rightarrow \mathbf{R}^{+}$corresponding to the homomorphism $P \rightarrow \mathbf{R}^{+}$given by $\Delta_{P}$, the modular function of $P$. Clearly $r: G / P \times \Gamma \rightarrow \mathbf{R}^{+}$is just $\alpha \mid G / P \times \Gamma$. Now the $\Gamma$ action on $G / P \times \Gamma \rightarrow \mathbf{R}^{+}$that appears in the construction of the 
Mackey range is just the restriction to $\Gamma$ of the $G$-action $G / P \times{ }_{\alpha} \mathbf{R}^{+}$. Since $\Delta$ : $P \rightarrow \mathbf{R}^{+}$is surjective, as is well known, this is the $G$-action $G / P \times{ }_{\alpha} P / \operatorname{ker} \Delta_{P}$ on which $G$ acts transitively with stabilizer $\operatorname{ker} \Delta_{P}$. Thus as a $\Gamma$-space, $G / P \times{ }_{r} \mathbf{R}^{+}$is just the action of $\Gamma$ on $G / \operatorname{ker} \Delta_{P}$. Since ker $\Delta_{P}$ is not compact, by Moore's ergodicity theorem $(\$ 2) \Gamma$ is ergodic on this space. Thus there is only one ergodic component, and so the modular flow is the action of $\mathbf{R}^{+}$on a point. This computation can clearly be carried out on any semisimple noncompact Lie group. If $\Gamma$ is a lattice in such a group $G$, and $P \subset G$ is a minimal parabolic, then the modular flow of the action of $\Gamma$ on $G / P$ will be the action of $\mathbf{R}^{+}$on a point.

The Dye-Krieger theorems were extended over the years by a number of persons to include within its framework actions of larger classes of groups. (In fact Dye did not restrict himself to the integers.) This work has recently culminated with the following theorems.

Theorem 5.15 (Connes-Feldman-Ornstein-Weiss [8, 37]). (i) $A$ free properly ergodic action of a discrete group is amenable if and only if it is orbit equivalent to a $\mathbf{Z}$-action.

(ii) The Dye-Krieger Theorems 5.11-5.13 hold for the class of amenable properly ergodic actions of discrete groups.

Theorem 5.16 (Connes-Feldman-Ornstein-Weiss $[8,37])$. (i) $A$ free properly ergodic action of a continuous group is amenable if and only if it is orbit equivalent to an $\mathbf{R}$-action.

(ii) For such actions, the modular flow is a complete invariant of orbit equivalence. In particular, any two free properly ergodic actions of continuous amenable unimodular groups with invariant measure are orbit equivalent.

EXAMPLE 5.17. If $G$ is a semisimple noncompact Lie group, $\Gamma \subset G$ a lattice, $P \subset G$ a minimal parabolic, we saw in Example 5.14 that the modular flow of $\Gamma$ on $G / P$ is independent of $G$ and $\Gamma$. Since these actions are amenable, Theorem 5.15 says that they are all orbit equivalent.

6. Rigidity: The Mostow-Margulis theorem and a generalization to ergodic actions. In this lecture we shall describe the proof of the Mostow-Margulis rigidity theorem for lattices in semisimple Lie groups and indicate how this result can be extended to yield results about orbit equivalence for ergodic actions of semisimple groups and their lattices.

Theorem 6.1 (Mostow-MARgulis RIGIDITY). Let $G, G^{\prime}$ be connected semisimple Lie groups with finite center, no compact factors, and $\Gamma, \Gamma^{\prime}$ irreducible lattices in $G, G^{\prime}$ respectively. Suppose $\mathbf{R}-\operatorname{rank}(G) \geqslant 2$. Then

(i) if $\Gamma$ and $\Gamma^{\prime}$ are isomorphic, then $G$ and $G^{\prime}$ are locally isomorphic.

(ii) In the center free case, any isomorphism $\Gamma \rightarrow \Gamma^{\prime}$ extends to a rational isomorphism $G \rightarrow G^{\prime}$.

This was first proved for cocompact lattices by Mostow [36] and for noncocompact lattices by Margulis [27]. In an extraordinary and highly original and innovative paper, Margulis then gave an alternate proof in [28] 
which subsumed both cases, gave stronger results on the extension of homomorphisms from $\Gamma$ to $G^{\prime}$, and which was powerful enough to prove the arithmeticity of lattices. In [55] we showed how Margulis' techniques could be incorporated into a proof of rigidity for ergodic actions. Theorem 6.1 is also true without the R-rank assumption as long as $G \neq \operatorname{PSL}(2, \mathbf{R})$. This is due to Mostow [36] and Prasad [39]. We now describe rigidity for ergodic actions.

Definition 6.2 [55]. Suppose $G$ is a semisimple connected Lie group with finite center and no compact factors. An ergodic $G$-space $S$ is called irreducible if every noncentral normal subgroup of $G$ is also ergodic on $S$. (Thus if $\Gamma$ is a lattice, $G / \Gamma$ is irreducible if and only if $\Gamma$ is irreducible.)

THEOREM 6.3 [55] (RIGIDITY FOR ERGODIC ACTIONS). Let $G, G^{\prime}$ be connected semisimple Lie groups with finite center and no compact factors, $S, S^{\prime}$ free irreducible ergodic $G$-, $G^{\prime}$-spaces, respectively, with finite invariant measure. Let $\mathbf{R}-\operatorname{rank}(G) \geqslant 2$. Suppose the actions are orbit equivalent. Then

(i) $G$ and $G^{\prime}$ are locally isomorphic,

(ii) in the centerfree case, we can take $G=G^{\prime}$, and then the actions on $S$ and $S^{\prime}$ are automorphically conjugate.

Thus, this theorem asserts that one has behavior that is diametrically opposed to the behavior of actions of amenable groups. Although Theorems 6.1 and 6.3 look rather different, let us show that they are both direct consequences of the following theorem.

THEOREM 6.4 [55]. Let $G, G^{\prime}$ be connected semisimple Lie groups with trivial center and no compact factors, and let $S$ be an irreducible ergodic G-space with finite invariant measure. Assume R-rank $(G) \geqslant 2$. Let $\alpha: S \times G \rightarrow G^{\prime}$ be a cocycle whose Mackey range is Zariski dense in $G^{\prime}$. Then $\alpha$ is equivalent to a cocycle $\beta$ that is the restriction of a rational epimorphism $\pi: G \rightarrow G^{\prime}$.

To deduce Theorem 6.1 from Theorem 6.4, one observes that when applied to the case $S=G / \Gamma$ and $\alpha: G / \Gamma \times G \rightarrow G^{\prime}$ a cocycle corresponding to a homomorphism $\Gamma \rightarrow G^{\prime}$, Theorem 6.4 yields the following theorem of Margulis.

Theorem 6.5 (MARgulis). $G, G^{\prime}$ as in Theorem 6.4, (R-rank $(G) \geqslant 2$ ), $\Gamma \subset G$ an irreducible lattice. Suppose $\pi \Gamma \rightarrow G^{\prime}$ is a homomorphism with $\pi(\Gamma)$ Zariski dense in $G^{\prime}$. Then $\pi$ extends to a rational epimorphism $G \rightarrow G^{\prime}$.

Theorem 6.1 then follows. To deduce Theorem 6.3 from Theorem 6.4, one simply applies Theorem 6.4 to the cocycle $\alpha: S \times G \rightarrow G^{\prime}$ coming from an orbit equivalence. The hypotheses of Theorem 6.4 are satisfied since the Mackey range of $\alpha$ is the $G^{\prime}$-space $S^{\prime}$ and the Borel density theorem implies Zariski density. The conclusion of 6.4 implies that of 6.3 by Proposition 3.3.

Let us give an example of how to apply Theorem 6.3 to some natural examples.

COROLlaRY 6.6 [55]. Let $G, G^{\prime}$ be connected simple noncompact Lie groups with finite center, $\Gamma, \Gamma^{\prime} \subset G, G^{\prime}$ lattices and suppose $S, S^{\prime}$ are free ergodic $\Gamma$-, 
$\Gamma^{\prime}$-spaces with finite invariant measure. Suppose $\mathbf{R}-\operatorname{rank}(G) \geqslant 2$, and that the $\Gamma$-action on $S$ and $\Gamma^{\prime}$-action on $S^{\prime}$ are orbit equivalent. Then $G$ and $G^{\prime}$ are locally isomorphic.

Proof. Let $X=\operatorname{ind}_{\Gamma}^{G}(S), X^{\prime}=\operatorname{ind}_{\Gamma^{\prime}}^{G^{\prime}}\left(S^{\prime}\right)$. Then one easily checks that the hypothesis of Theorem 6.3 is satisfied.

EXAMPLE 6.7 [55]. As we vary $n, n \geqslant 2$, the natural actions of $\operatorname{SL}(n, Z)$ on $\mathbf{R}^{n} / Z^{n}$ by automorphisms are mutually nonorbit equivalent.

We now turn to some proofs. We will not prove Theorem 6.4 here, but rather only Theorem 6.5 (which therefore gives us a proof of Theorem 6.1). The first part of the proof we present is different from Margulis' original argument. Instead, we present an argument which generalizes nicely when one attempts to prove Theorem 6.4. It is perhaps also more transparent than the original argument. The second part of the proof will be that of Margulis, although we shall try to give some motivation. For the proof of Theorem 6.4, see [55].

Proof of Theorem 6.5. Let $P \subset G, P^{\prime} \subset G^{\prime}$ be minimal parabolic subgroups. We have a homomorphism $\pi: \Gamma \rightarrow G^{\prime}$ and hence $G^{\prime} / P^{\prime}$ becomes a compact metrizable $\Gamma$-space. On the other hand, as we observed in Example 5.4 , the action of $\Gamma$ on $G / P$ is ergodic and amenable. By the remarks following Definition 5.2, there is a measurable $\Gamma$-map $\varphi: G / P \rightarrow M\left(G^{\prime} / P^{\prime}\right)$, the latter space being the space of probability measures on $G^{\prime} / P^{\prime}$. By Theorem 5.7 , the action of $G^{\prime}$ on $M\left(G^{\prime} / P^{\prime}\right)$ is smooth, so $\hat{M}\left(G^{\prime} / P^{\prime}\right)=\left[M\left(G^{\prime} / P^{\prime}\right)\right] / G^{\prime}$ is countably separated and generated. Since $\varphi$ is a $\Gamma$-map, $\varphi(x \gamma)=\varphi(x) \pi(\gamma)$, so $\varphi(x \gamma) \equiv \varphi(x)$ in $\hat{M}\left(G^{\prime} / P^{\prime}\right)$. By ergodicity of $\Gamma$ on $G / P$, the projection of $\varphi$ into $\hat{M}\left(G^{\prime} / P^{\prime}\right)$ is essentially constant, i.e. $\varphi(G / P)$ can be assumed to lie in one $G^{\prime}$-orbit in $M\left(G^{\prime} / P^{\prime}\right)$. Thus, we can view $\varphi$ as a $\Gamma$-map $\varphi: G / P \rightarrow G^{\prime} / H^{\prime}$, and by Theorem $5.8, H^{\prime}$ is an amenable algebraic subgroup. What we have done is to obtain a $\Gamma$-map $\varphi$ where the image is no longer an infinite dimensional space $M\left(G^{\prime} / P^{\prime}\right)$ but an algebraic variety $G^{\prime} / H^{\prime}$. The existence of such a measurable map $\varphi$ is the first main step in the proof. The second step is the following fundamental lemma of Margulis.

Lemma A. $\varphi: G / P \rightarrow G^{\prime} / H^{\prime}$ is a rational mapping of algebraic varieties.

Let us show why this lemma suffices to prove the theorem. Suppose $\varphi$ : $G / P \rightarrow G^{\prime} / H^{\prime}$ is a rational mapping such that $\varphi(x \gamma)=\varphi(x) \pi(\gamma)$. Let $R\left(G / P, G^{\prime} / H^{\prime}\right)$ be the space of rational mappings. Then $G \times G^{\prime}$ acts on $R\left(G / P, G^{\prime} / H^{\prime}\right)$ by

$$
\left[\left(g, g^{\prime}\right) \cdot f\right](x)=f(x g) \cdot\left(g^{\prime}\right)^{-1} \text {. }
$$

The fact that $\varphi$ is a $\Gamma$-map means $\varphi$ is fixed under $\Gamma$, where $\Gamma$ is identified with the subgroup of $G \times G^{\prime}$ given by $\{(\gamma, \pi(\gamma))\}$. Let $\bar{\Gamma}$ be the algebraic hull of $\Gamma$ in $G \times G^{\prime}$. Then $\varphi$ is also fixed under $\bar{\Gamma}$. We claim that $\bar{\Gamma}$ is the graph of homomorphism $G \rightarrow G^{\prime}$. Since $\Gamma$ is Zariski dense in $G, \bar{\Gamma}$ must project onto all of $G$. So suppose $\left(g, h_{1}\right),\left(g, h_{2}\right) \in \bar{\Gamma}$. Then $\varphi(x g)=\varphi(x) h_{1}$ and $\varphi(x g)=$ $\varphi(x) h_{2}$. Therefore $h_{1} h_{2}^{-1}$ leaves $\varphi(G / P)$ pointwise fixed. But $\pi(\Gamma)$ leaves $\varphi(G / P)$ invariant, and since $\pi(\Gamma)$ is Zariski dense in $G^{\prime}, \varphi(G / P)$ must be Zariski dense in $G^{\prime} / H^{\prime}$. Therefore $h_{1} h_{2}^{-1}$ leaves all $G^{\prime} / H^{\prime}$ pointwise fixed, and 
since $\bigcap_{g \in G^{\prime}} g H^{\prime} g^{-1}=\{e\}$ (since it is an amenable normal subgroup), $h_{1} h_{2}^{-1}=$ $e$. Therefore $\bar{\Gamma}$ is the graph of a function $G \rightarrow G^{\prime}$, which is a homomorphism since $\Gamma \subset G$ is Zariski dense and the map is a homomorphism on $\Gamma$.

We now return to the proof of the lemma. We must show that a certain measurable mapping between varieties is actually rational. There is one well known situation in which a measurable map is known to have much stronger properties, namely if the map is a homomorphism. For example, any measurable homomorphism between Lie groups is $C^{\infty}$, and similarly, any measurable homomorphism between real algebraic groups $R \rightarrow R^{\prime}$, with $R$ reductive, will be rational on all unipotent subgroups of $R$. Of course our map $\varphi$ is defined on $G / P$ which is not a group. However, up to a set of measure 0 , it is a group. For example, consider $G=\operatorname{SL}(n, \mathbf{R}), P=$ upper triangular subgroup. Let $U$ be the lower triangular unipotent matrices. Then the natural map $G \rightarrow G / P$ carries $U$ onto an open subset of measure 1. Furthermore, this establishes an isomorphism of $U$ with its image as algebraic varieties. Thus, we can view $\varphi$ as a map $U \rightarrow G^{\prime} / H^{\prime}$. Now, although we have $\varphi$ defined on a group, it is not a homomorphism (as the image is not even a group). We do however, have some sort of algebraic relation, namely the fact that $\varphi$ is a $\Gamma$-map. Thus we might hope to be able to force this algebraic relation to show that $\varphi$ only depends upon a homomorphism of $U$. This, however, is not possible. As we shall see, when we try to force the algebra, we shall need some commutativity of $U$ with elements of $A$, where $A$ is the positive diagonals. But the centralizer of $U$ in $A$ is trivial. In the R-rank 1 case, we can proceed no further, but in higher rank all is not lost. Let us fix an element $t \in A$, and consider the centralizer $C_{t}$. For example, in $\operatorname{SL}(3, \mathbf{R})$, let

$$
t=\left(\begin{array}{lll}
\lambda & 0 & 0 \\
0 & \lambda & 0 \\
0 & 0 & \lambda^{-2}
\end{array}\right), \quad C_{t}=\left\{\left(\frac{M}{0} \frac{0}{\alpha}\right) \text { where } \alpha=(\operatorname{det} M)^{-1}\right\} .
$$

Let

$$
C_{t}^{u}=C_{t} \cap U=\left\{\left(\begin{array}{lll}
1 & 0 & 0 \\
a & 1 & 0 \\
0 & 0 & 1
\end{array}\right)\right\} .
$$

Now $U \cong \mathbf{R}^{3}$, and $C_{t}^{u} \cong \mathbf{R}$. Thus $C_{t}^{u}$ will give us one direction in $U$, and $C_{t}$ is a reductive group that has a centralizer in $A$. As we shall see, this will be enough to show that $\varphi: U \rightarrow G^{\prime} / H^{\prime}$ depends rationally on $C_{t}^{u}$. But now if we vary $t \in A$, we can pick up the other directions in the same way. The following lemma of Margulis is now clearly relevant.

Lemma B. Let $\varphi$ be a measurable function defined on $\mathbf{R}^{n} \times \mathbf{R}^{k}$. If $\varphi$ is rational in $x$ for almost all $y \in \mathbf{R}^{k}$ and rational in $y$ for almost all $x \in \mathbf{R}^{n}$, then $\varphi$ is rational.

The above remarks about $\operatorname{SL}(n, \mathbf{R})$ extend to general $G$. Thus, if we let $U$ be the unipotent radical of the parabolic opposite to $P$, then $U \rightarrow G / P$ is an isomorphism of algebraic varieties with its image, the latter being open and of full measure. Let $A \subset P$ be a maximal abelian R-diagonalizable subgroup and 
$t \in A, t \neq 0$. Let $C_{t}$ be the centralizer of $t$ in $G$. Then $C_{t}$ is reductive. Letting $C_{t}^{u}=U \cap C_{t}, U$ can be built from the various $C_{t}^{u}$ by varying $t$. Thus, using Lemma $\mathrm{B}$, it suffices now to prove the following. View $\varphi: G / P \rightarrow G^{\prime} / H^{\prime}$ as a map $\varphi: G \rightarrow G^{\prime} / H^{\prime}$, with $\varphi(p g)=\varphi(g)$, for $p \in P$.

Lemma C. For almost all $g \in G, \varphi(c g)$ depends rationally on $c$ for $c \in C_{t}^{u}$ ( for any $t \in A$ ).

Proof. Let $C_{t}=C$. We want to study dependence of $\varphi$ on $C$, so for each $g \in G$, define $\omega_{g}: C \rightarrow G^{\prime} / H^{\prime}$ by $\omega_{g}(c)=\varphi(c g)$.

Thus we have a map $\omega: G \rightarrow F\left(C, G^{\prime} / H^{\prime}\right)$, the latter being the space of measurable maps $C \rightarrow G^{\prime} / H^{\prime}$. Let $T=\left\{t^{n}\right\}$. Then $\omega_{t g}(c)=\omega(c t g)=\omega(t c g)$ $=\omega(c g)$ (since $t \in P$ ), and so we have $\omega_{t g}(c)=\omega_{g}(c)$. Thus we can view $\omega$ as a map $\omega: G / T \rightarrow F\left(C, G^{\prime} / H^{\prime}\right)$. We now use $\Gamma$-invariance of $\varphi$ :

$$
\omega_{g \gamma}(c)=\varphi(c g \gamma)=\varphi(c g) \pi(\gamma)=\omega_{g}(c) \cdot \pi(\gamma) .
$$

Thus, $\omega_{g \gamma}$ and $\omega_{g}$ are in the same $G^{\prime}$-orbit in $F\left(C, G^{\prime} / H^{\prime}\right)$, where $G^{\prime}$ acts on the latter pointwise. We now need another smoothness result.

Lemma D. Every $G^{\prime}$-orbit in $F\left(X, G^{\prime} / H^{\prime}\right)$ is locally closed, where $X$ is a measure space.

We observe that if $X$ is finite, this is immediate from the fact that $F\left(X, G^{\prime} / H^{\prime}\right)$ would then be a variety. Margulis observed that the lemma is true for any measure space $X$. For a simple proof, see the appendix of [55].

Returning now to the proof of Lemma C, we have that $\omega_{g \gamma} \equiv \omega_{g}$ when projected to $\left[F\left(C, G^{\prime} / H^{\prime}\right)\right] / G^{\prime}$. By Lemma $\mathrm{D}$, this latter space is countably generated and separated, and by Moore's theorem $\Gamma$ is ergodic on $G / T$. Therefore, all $\omega_{g}$ are equal when projected to $\left[F\left(C, G^{\prime} / H^{\prime}\right)\right] / G^{\prime}$, or equivalently, all $\omega_{g}$ lie in the same $G^{\prime}$-orbit. So for $a, g \in G$, we have

$$
\omega_{a g}=\omega_{g} \cdot h(a, g)
$$

where $h(a, g) \in G^{\prime}$ and $h$ is measurable. For any $f \in F\left(C, G^{\prime} / H^{\prime}\right)$, let $G_{f}^{\prime}$ be the stabilizer, and $N_{f}$ the normalizer of $G_{f}^{\prime}$ in $G^{\prime}$. Clearly for $a \in C, G_{\omega_{a g}}^{\prime}=G_{\omega_{g}}^{\prime}$, so for $a \in C, h(a, g) \in N_{\omega_{g}}$. Suppose now that $a_{1}, a_{2} \in C$. Then

$$
\begin{aligned}
\omega_{g}(c) \cdot h\left(a_{1} a_{2}, g\right) & =\omega_{a_{1} a_{2} g}(c)=\varphi\left(c a_{1} a_{2} g\right) \\
& =\omega_{a_{2} g}\left(c a_{1}\right)=\omega_{g}\left(c a_{1}\right) \cdot h\left(a_{2}, g\right) \\
& =\varphi\left(c a_{1} g\right) \cdot h\left(a_{2}, g\right)=\omega_{a_{1} g}(c) \cdot h\left(a_{2}, g\right) \\
& =\omega_{g}(c) \cdot h\left(a_{1}, g\right) h\left(a_{2}, g\right) .
\end{aligned}
$$

Thus, for almost all $g, a \rightarrow h(a, g)$ is a measurable homomorphism

$$
C \rightarrow N_{\omega_{g}} / G_{\omega_{g}}^{\prime}
$$

We have $\varphi(c a g)=\varphi(c g) \cdot h(a, g)$. Thus for $a$ in any unipotent subgroup of $C, a \rightarrow \varphi(c a g)$ depends rationally on $a$. Choosing this subgroup to be $c^{-1} C_{t}^{u} c$, we obtain $\varphi(b g)$ depends rationally on $b \in C_{t}^{u}$. This completes the proof. 
7. Complements to the rigidity theorem for ergodic actions: Foliations by symmetric spaces and Kazhdan's property $(T)$. The rigidity theorem for ergodic actions stated in $\$ 6$ allowed us to distinguish ergodic actions of lattices on the basis of orbit equivalence if the actions had finite invariant measure (e.g. Corollary 6.6 and Example 6.7). However, some of the most interesting actions of lattices, e.g., the action of $\operatorname{SL}(n, Z)$ on $\mathbf{P}^{n-1}$ or other flag and Grassman varieties, do not have finite invariant measure. We now indicate how to extend the rigidity theorem to enable us to deal with this situation. The main step is to first extend the rigidity theorem to actions of general connected groups.

Let $H$ be a connected group. Every locally compact group has a unique maximal normal amenable subgroup $N$. If $H$ is connected $H / N$ will be a product of noncompact connected simple Lie groups with trivial center. We shall say that an ergodic action of $H$ is irreducible if the inverse image in $H$ of each of these simple factors of $H / N$ is still ergodic.

THEOREM 7.1 [56]. Let $H, H^{\prime}$ be connected locally compact groups, $N, N^{\prime}$ the maximal normal amenable subgroups. Suppose R-rank $(H / N) \geqslant 2$. Let $S, S^{\prime}$ be free ergodic irreducible $H$-, $H^{\prime}$-spaces with finite invariant measure, and suppose the actions are orbit equivalent. Then $H / N$ and $H^{\prime} / N^{\prime}$ are isomorphic, and $N$ is compact if and only if $N^{\prime}$ is also compact.

Thus, for connected groups, orbit equivalence implies isomorphism of the semisimple parts of the groups. The proof of this result is an extension of the proof of the rigidity theorem for ergodic actions of semisimple groups. To see how to apply this to obtain results about actions of lattices without invariant measure, observe that the orbit space of $\Gamma$ acting on $G / H$ can be identified with the orbit space of $H$ acting on $G / \Gamma$. Theorem 7.1 deals with the latter situation since now $G / \Gamma$ has a finite $H$-invariant measure. so we can try to apply this to the action of $\Gamma$ on $G / H$. One can then prove the following precise result.

THEOREM 7.2 [56]. Let $G, G^{\prime}$ be connected semisimple Lie groups with finite center, $\Gamma, \Gamma^{\prime}$ irreducible lattices. Let $H \subset G, H^{\prime} \subset G^{\prime}$ be almost connected noncompact subgroups. Assume the actions of $\Gamma$ on $G / H$ and $\Gamma^{\prime}$ on $G^{\prime} / H^{\prime}$ are essentially free and orbit equivalent. Let $N, N^{\prime} \subset H, H^{\prime}$ be the maximal normal amenable subgroups, and suppose $\mathbf{R}-\operatorname{rank}(H / N) \geqslant 2$. Then $H / N$ and $H^{\prime} / N^{\prime}$ are locally isomorphic.

EXAMPLE 7.3 [56]. As we vary $n, n \geqslant 2$, the actions of $\operatorname{SL}(n, Z)$ on $\mathbf{P}^{n-1}$ are mutually nonorbit equivalent. This follows by simply observing that the semisimple parts of the corresponding maximal parabolics in $\operatorname{SL}(n, \mathbf{R})$ are not isomorphic. (Actually, Theorem 7.2 will not apply to compare the cases $n=2$ and $n=3$. However, the action of $\operatorname{SL}(2, Z)$ on $\mathbf{P}^{1}$ is amenable, while the action of $\operatorname{SL}(3, Z)$ on $\mathbf{P}^{2}$ is not.) In a similar fashion, one can read off a large number of results about actions of lattices on the flag and Grassman varieties.

A natural question that arises in light of Theorem 7.1 is how sensitive orbit equivalence is to the way in which $H$ is built from $N$ and $H / N$. For example, what is the relation of actions of $\operatorname{SL}(n, \mathbf{R}) \times \mathbf{R}^{n}$ to that of $\operatorname{SL}(n, \mathbf{R}) \subseteq \mathbf{R}^{n}$, 
where the latter semidirect product just results from the natural action of $\operatorname{SL}(n, \mathbf{R})$ on $\mathbf{R}^{n}$ ? To answer this question, we recall Kazhdan's notion of property $(\mathrm{T})$ for groups, and then indicate how to define this for actions.

Let $G$ be a locally compact group, and $I$ the one dimensional trivial representation. If $\pi_{n}, \pi$ are unitary representations of $G$, then recall $\pi_{n} \rightarrow \pi$ means that for any unit vectors $v_{1}, \ldots, v_{k} \in H_{\pi}$ there exist unit vectors $v_{1}^{n}, \ldots, v_{k}^{n} \in H_{\pi_{n}}$ such that $\left\langle\pi_{n}(g) v_{i}^{n} \mid v_{j}^{n}\right\rangle \rightarrow\left\langle\pi(g) v_{i} \mid v_{j}\right\rangle$ uniformly on compact sets in $G$ for each $i, j$. Kazhdan [24] defined a group to have property (T) if $\pi_{n} \rightarrow I$ implies $I \leqslant \pi_{n}$ for $n$ sufficiently large.

THEOREM 7.4 (KAZHDAN [24, 9]). (i) Semisimple Lie groups with all simple factors having R-rank at least 2 have property (T). (Actually, Kazhdan proved this assuming $\mathbf{R}$-rank $\geqslant 3$. That one only need assume $\mathbf{R}$-rank $\geqslant 2$ was observed by a number of persons, e.g. [9].)

(ii) Any lattice subgroup of a group with property (T) also has property (T).

We shall also need the following result of Wang.

TheOREM 7.5 (WANG [46]). $\operatorname{SL}(n, \mathbf{R})\left(S \mathbf{R}^{n}\right.$ has property $(\mathrm{T})$, and hence so does $\operatorname{SL}(n, Z) \odot Z^{n}(n \geqslant 3)$.

We now define property $(\mathrm{T})$ for ergodic actions. For simplicity, we restrict attention to actions of discrete groups. This notion for actions originally appeared in [57].

Let $G$ be a discrete group, $S$ an ergodic $G$-space. Let $\alpha: S \times G \rightarrow U(H)$ be a unitary group valued cocycle. Let $v, w: S \rightarrow H$ be Borel functions with $\|v\|_{\infty}$ $=\|w\|_{\infty}=1$. Let $f_{\alpha, v, w}: S \times G \rightarrow \mathbf{C}$ be given by

$$
f_{\alpha, v, w}(s, g)=\langle\alpha(s, g) v(s g) \mid w(s)\rangle \text {. }
$$

We consider $f_{\alpha, v, w}$ as a function $G \rightarrow F(S, \mathbf{C})$, and we endow $F(S, \mathbf{C})$ with the topology of convergence in measure. For cocycles $\alpha_{n}, \alpha$, we say $\alpha_{n} \rightarrow \alpha$ if given $v_{1}, \ldots, v_{k}: S \rightarrow H_{\alpha},\left\|v_{i}\right\|_{\infty}=1$, there exist $v_{1}^{n}, \ldots, v_{k}^{n}: S \rightarrow H_{\alpha_{n}}$ such that $f_{\alpha_{n}, v_{l}^{n}, v_{j}^{n}}$ $\rightarrow f_{\alpha, v_{t}, v}$, pointwise on $G$ (i.e. in measure on $S$ for each $g \in G$ ) for all $i, j$.

DEFINITION 7.6 [57]. The action of $G$ on $S$ has property (T) if $\alpha_{n} \rightarrow I$ implies $\alpha_{n} \geqslant I$ for $n$ sufficiently large. Here $I$ is the one dimensional trivial cocycle and $\alpha \geqslant I$ means $\alpha \sim \beta$ where $\beta(s, g) v=v$ for some nonzero vector $v$.

We then have the following results.

THEOREM 7.7 [57]. (a) If $G$ has property (T), and $S$ has a finite invariant measure then $S$ has property $(\mathrm{T})$.

(b) If $S$ has property (T), finite invariant measure and is weak mixing (i.e. there are no finite dimensional invariant subspaces in $L^{2}(S) \ominus \mathbf{C}$ ), then $G$ has property (T).

(c) For free actions of discrete groups, property (T) is an invariant of orbit equivalence.

Combining 7.5 and 7.7, we have the following, showing that, in fact, orbit equivalence is quite sensitive to the way $H$ is constructed from $N$ and $H / N$. 
COROllaRy 7.8 [57]. Let $n \geqslant 3$, and $\Gamma_{1}=\operatorname{SL}(n, Z) \times Z^{n}, \quad \Gamma_{2}=$ $\operatorname{SL}(n, Z)(S) Z^{n}$. Then $\Gamma_{1}$ and $\Gamma_{2}$ do not have free orbit equivalent weakly mixing actions with finite invariant measure.

We shall now describe a geometric interpretation of the rigidity theorem for ergodic actions. We begin by recalling the geometric formulation of the Mostow-Margulis theorem. Let $G$ be a connected semisimple Lie group with finite center and no compact factors, $K \subset G$ a maximal compact subgroup, and $\Gamma \subset G$ a torsion free lattice. Then $G / K$ is a Riemannian symmetric space (diffeomorphic to Euclidean space), and $\Gamma$ operates properly discontinuously on $X=G / K$. Thus $\Gamma \backslash X$ is a locally symmetric space of finite volume, and $\pi_{1}(\Gamma \backslash X) \cong \Gamma$.

Theorem 7.9 (Mostow-MARgulis Rigidity, GeOMETRIC FORM). Let $M_{1}, M_{2}$ be locally symmetric Riemannian manifolds of finite volume whose universal covers, $X_{1}, X_{2}$ are symmetric spaces of purely noncompact type, and whose fundamental groups $\pi_{1}\left(M_{i}\right)$ act as irreducible groups of isometries of $X_{i}$. Suppose further that the rank of $M_{1}$ is at least 2 . Then any isomorphism $\pi_{1}\left(M_{1}\right) \rightarrow \pi_{1}\left(M_{2}\right)$ is induced by a diffeomorphism $M_{1} \rightarrow M_{2}$ that is an isometry modulo normalizing scalar multiples.

Rougly speaking, this asserts that for a particular class of Riemannian manifolds, i.e. suitable locally symmetric spaces, that a purely topological invariant, namely the fundamental group, determines the Riemannian structure. We now describe an analogous geometric interpretation of the rigidity theorem for actions which will make an assertion about foliations by symmetric spaces.

Let $G$ be a connected semisimple noncompact Lie group with finite center and no compact factors, $K \subset G$ a maximal compact subgroup, and $(S, \mu)$ a free ergodic $G$-space with finite invariant measure. Let $Y=S / K$. Then because $K$ is compact, $Y$ is a standard Borel space, and the orbits in $S$ yield an equivalence relation $\mathcal{T}$ on $Y$ in which each equivalence class can be identified with $G / K$. Thus, $(Y, \mathcal{T})$ is a "Riemannian measurable foliation", i.e., a measure space with an equivalence relation $\mathcal{T}$ in which each equivalence class (or "leaf") has the structure of a $C^{\infty}$-Riemannian manifold, so that these structures vary measurably in a suitable sense [53] over $Y$. Given $Y, Y^{\prime}$, two spaces supporting Riemannian measurable foliations, we call them isometric if there is a measure space isomorphism between $Y$ and $Y^{\prime}$ that carries leaves onto leaves isometrically (possibly after discarding null sets of leaves). By a transversal for $(Y, \mathcal{T})$ we mean a Borel set intersecting almost every leaf in a countable set. Such a set $T$ will have a natural equivalence relation on it with countable equivalence classes (namely $\mathcal{T} \mid T$ ), and a natural measure class $\nu$ satisfying the condition that for $B \subset T, \nu(B)=0$ if and only if the union of the leaves intersecting $B$ has $\mu$-measure $0[13,41]$. We call two Riemannian measurable foliations transversally equivalent if they have isomorphic transversals. By an isomorphism of transversals. we mean isomorphism as measure 
spaces with equivalence relations, i.e., a measure space isomorphism carrying one equivalence relation onto the other. This is a purely measure-theoretic invariant of the foliation. The following is the geometric version of Theorem 6.3 .

THEOREM 7.10 [55] (RIGIDITY FOR FOLIATIONS BY SYMMETRIC SPACES). Let $G$, $G^{\prime}, S, S^{\prime}$ be as in Theorem 6.3. Let $Y=S / K, Y^{\prime}=S^{\prime} / K^{\prime}$ where $K, K^{\prime} \subset G, G^{\prime}$ are the maximal compact subgroups. Let $(Y, \mathcal{T}),\left(Y^{\prime}, \mathcal{T}^{\prime}\right)$ be the associated Riemannian measurable foliations by symmetric spaces. If the foliations are transversally equivalent, then they are isometric, modulo normalizing scalar multiples (independent of the leaves).

Thus, roughly speaking, for suitable foliations in which the leaves are symmetric spaces, a purely measure-theoretic invariant, namely the measure theory of the transversal, determines the Riemannian structure on almost every leaf.

As we have already remarked, the rigidity theorem for lattices holds in the $\mathbf{R}$-rank 1 case as well as long as $G \neq \operatorname{PSL}(2, \mathbf{R})$, although the proof we have given in $\$ 6$ does not apply, and one must use other techniques, for example those of Mostow [36] and Prasad [39]. It is natural to inquire as to what extent the rigidity theorem for ergodic actions holds in the R-rank 1 case as well. In [58] we proved the following result in this direction, applying basic results of Mostow [35] on quasi-conformal mappings.

THeOREM 7.11 [58]. Let $S, S^{\prime}$ be free ergodic $\mathrm{SO}(1, n) /\{ \pm, I\}$-spaces with finite invariant measure, and assume $n \geqslant 3$. Let $(Y, \mathcal{T}),\left(Y^{\prime}, \mathcal{J}^{\prime}\right)$ be the associated measurable foliations by hyperbolic space (as in the discussion preceding Theorem 7.10). If $(Y, \mathcal{T})$ and $\left(Y^{\prime}, \mathcal{T}^{\prime}\right)$ are quasi-conformally equivalent, then they are isometric (modulo a normalizing scalar independent of the leaf), and the actions of $\mathrm{SO}(1, n) /\{ \pm I\}$ on $S$ and $S^{\prime}$ are automorphically conjugate.

Here, of course, quasi-conformal equivalence asserts the existence of a measure space isomorphism taking (almost all) leaves to leaves quasiconformally. We remark that the analogous statement for $\mathbf{R}^{n}$ actions can be shown to be false by many counterexamples.

8. Margulis' finiteness theorem. In $\S 6$, we saw how the analysis of the ergodic action of $\Gamma$ on $G / P$ led to Margulis' proof of the rigidity theorem. Margulis has also demonstrated some other deep properties of this ergodic action and used this to obtain very strong results about the structure of $\Gamma$. More precisely, he has shown the following.

TheOREM 8.1 (MARGulis [30, 31]). Let $G$ be a connected semisimple Lie group with finite center and no compact factors, and assume $\mathbf{R}$-rank $(G) \geqslant 2$. Let $\Gamma \subset G$ be an irreducible lattice, and $H=\Gamma / N$ a nonamenable quotient group. Then $N \subset Z / Z(G)$, the center of $G$, and in particular, is finite.

If we further assume that the R-rank of every simple factor of $G$ is at least 2, then $\Gamma$ has property (T) of Kazhdan [24], and hence if $H=\Gamma / N$ is an 
amenable quotient, $H$ must also have property (T) and hence is finite. Thus, we conclude the following.

COROLlARY 8.2. Let $G$ be a connected semisimple Lie group with finite center and assume R-rank of each simple factor of $G$ is at least 2. Let $\Gamma \subset G$ be an irreducible lattice. Then every normal subgroup of $G$ is either finite or of finite index.

Margulis' results are in fact significantly more general, both in terms of taking lattices in products of algebraic groups defined over various local fields and in terms of rank restrictions. The basic difficult step in the proof of Theorem 8.1 is the following result concerning the action of $\Gamma$ on $G / P$. Let $P^{\prime}$ be another parabolic subgroup containing $P$. Then there is a $\Gamma$-map $G / P \rightarrow$ $G / P^{\prime}$, i.e. $G / P^{\prime}$ is a $\Gamma$-space factor of $G / P$.

Theorem 8.3 (MARgulis [30]). Let $G, \Gamma$ be as in Theorem 8.1, $P \subset G a$ minimal parabolic. Then any measurable factor of the $\Gamma$-space $G / P$ is of the form $G / P \rightarrow G / P^{\prime}$ for some parabolic $P^{\prime} \supset P$.

In other words, every measurable $\Gamma$-factor of $G / P$ is actually also a $G$-factor. This theorem is difficult and we will not prove it here. Instead, we show how to deduce Theorem 8.1 from it.

Let $H=\Gamma / N$ be a nonamenable quotient. Then there is a compact metric $H$-space $X$ so that there is no $H$-invariant measure on $X$. We can also view $X$ as a compact metric $\Gamma$-space. Since the action of $\Gamma$ on $G / P$ is amenable, by the discussion following Definition 5.2, there is a measurable $\Gamma$-map $\varphi: G / P \rightarrow$ $M(X)$, where the latter is the space of probability measures on $X$. If we let $\mu$ be a measure on $G / P$ in the natural measure class, then $\left(M(X), \varphi_{*}(\mu)\right)$ is a $\Gamma$-space factor of $G / P$. Thus, there is some parabolic $P^{\prime}$ so that as $\Gamma$-spaces, $\left(M(X), \varphi_{*}(\mu)\right)$ is conjugate to $G / P^{\prime}$. Since there are no fixed points in $M(X)$ under $\Gamma, P^{\prime} \neq G$. But $N$ acts trivially on $M(X)$ by definition, so $N$ is trivial on $G / P^{\prime}$ which implies $N \subset \bigcap_{g \in G} g P^{\prime} g^{-1}$, a proper normal subgroup of $G$. Dividing $G$ by its center, it clearly suffices to observe that if $\Gamma \subset \prod_{i \in I} G_{i}$ is an irreducible lattice in a product of simple Lie groups with trivial center, that $N=\Gamma \cap \prod_{i \in J} G_{i}$ is trivial for $J \subset I$ a proper subset. But since $N$ is normalized by $\Gamma$ and $\prod_{I-J} G_{i}$, it is normalized by the product of these groups which is dense in $G$ by irreducibility. The result follows.

9. Margulis' arithmeticity theorem. (This section will require a bit more knowledge about algebraic groups than previous sections. We also caution the reader that in this section, by algebraic group, Zariski closure, etc., we shall mean with respect to the algebraically closed field, unless we explicitly declare otherwise in a given instance.)

In this section we describe the proof of Margulis' arithmeticity theorem for lattices in semisimple Lie groups. The proof of the rigidity theorem in $\S 6$ was based on a result asserting that under suitable hypotheses, a homomorphism of $\Gamma$ into a real algebraic group extended to a homomorphism of $G$. This result is also basic to the proof of the arithmeticity theorem. However, we shall also 
need results concerning homomorphisms of $\Gamma$ into complex groups and algebraic groups over local fields. With some additional comments, the proof of Theorem 6.5 can be applied to give us these needed results, so that the bulk of the work of the proof of arithmeticity has in fact already been done. But before passing to these arguments, let us recall the statement of the problem.

The first example of a lattice in a Lie group is the integer lattice $Z^{n} \subset \mathbf{R}^{n}$. This is of course not the only lattice in $\mathbf{R}^{n}$. However if $L$ is any lattice there is an automorphism $A: \mathbf{R}^{n} \rightarrow \mathbf{R}^{n}$ such that $A\left(Z^{n}\right)=L$. Thus, $L$ is "arithmetically" defined.

To get other examples of lattices, suppose $G \subset \mathrm{GL}(n, \mathbf{C})$ is an algebraic group defined over $\mathbf{Q}$, i.e. there is an ideal $I \subset \mathbf{Q}\left[a_{i j}, \operatorname{det}\left(a_{i j}\right)^{-1}\right]$ such that $G=\{a \in \operatorname{GL}(n, \mathbf{C}) \mid p(a)=0$ for all $p \in I\}$. As usual, if $B \subset \mathbf{C}$ is any subring, we let

$$
G_{B}=\left\{a \in G \mid a_{i j} \in B \text {, for all } i, j \text { and } \operatorname{det}\left(a_{i j}\right)^{-1} \in B\right\} .
$$

TheOrem 9.1 (BOREL-HARISH-CHANDRA [5]). If $G$ is semisimple, then $G_{Z}$ is a lattice in $G_{\mathbf{R}}$.

For example, for $G=\operatorname{SL}(n, \mathbf{C})$, we have $\operatorname{SL}(n, Z)$ is a lattice in $\operatorname{SL}(n, \mathbf{R})$. The question the arithmeticity theorem answers is to what extent this is a general construction, i.e. to what extent are lattices arithmetically defined? We now exhibit two ways of modifying a given lattice to obtain a new lattice.

Definition 9.2. If $\Gamma, \Gamma^{\prime}$ are discrete groups, then $\Gamma$ and $\Gamma^{\prime}$ are called commensurable if $\left[\Gamma: \Gamma \cap \Gamma^{\prime}\right]<\infty$ and $\left[\Gamma^{\prime}: \Gamma \cap \Gamma^{\prime}\right]<\infty$.

Proposition 9.3. If $\Gamma, \Gamma^{\prime} \subset G, \Gamma$ is a lattice and $\Gamma, \Gamma^{\prime}$ are commensurable, then $\Gamma^{\prime}$ is a lattice.

For example, given that $\operatorname{SL}(n, Z)$ is a lattice, $\{a \in \operatorname{SL}(n, Z) \mid a=I \bmod p$ for a given prime $p\}$ is a commensurable lattice.

Here is another way to get new lattices.

Proposition 9.4. If $\Gamma \subset H$ is a lattice, $\varphi: H \rightarrow G$ a surjective homomorphism with compact kernel then $\varphi(\Gamma)$ is a lattice in $G$.

Margulis' theorem says that aside from these two types of rather trivial modifications, every lattice in a semisimple Lie group of higher R-rank arises as in Theorem 9.1. More precisely, let us make the following definition. (If $H$ is a group, $H^{0}$ denotes the topologically connected component of the identity.)

Definition 9.5. Let $G$ be a connected semisimple Lie group with trivial center and no compact factors. Let $\Gamma \subset G$ be a lattice. Then $\Gamma$ is called arithmetic if there exists an algebraic group $H$ defined over $\mathbf{Q}$, and a surjective homomorphism $\varphi: H_{\mathbf{R}}^{0} \rightarrow G$ such that

(i) $\operatorname{kernel}(\varphi)$ is compact;

(ii) $\varphi\left(H_{Z} \cap H_{\mathbf{R}}^{0}\right)$ is a lattice in $G$ commensurable with $\Gamma$.

Theorem 9.6 (Margulis [28]). Let $G$ be as in Definition 9.5, and assume $\mathbf{R}-\operatorname{rank}(G) \geqslant 2$. Then any irreducible lattice in $G$ is arithmetic. 
As we indicated above, the proof is based on two further results about homomorphisms of $\Gamma$.

TheOREM 9.7 (MARgulis [28]). Let $\Gamma \subset G$ be an irreducible lattice, $G$ as above, $\mathbf{R}-\operatorname{rank}(G) \geqslant 2$.

(i) If $I I$ is a (complex) simple algebraic group, connected and with trivial center, then any homomorphism $\pi: \Gamma \rightarrow H$ with $\pi(\Gamma)$ Zariski dense in $H$ either satisfies $\overline{\pi(\Gamma)}$ compact or extends to a rational endomorphism $\tilde{G} \rightarrow H$, where $\tilde{G}$ is the Zariski closure of $G$ (embedding $G$ in the linear transformations in the complexified Lie algebra for example).

(ii) Any homomorphism $\pi: \Gamma \rightarrow H_{K}$ where $H$ is a semisimple algebraic group over $K$, and $K$ is a local totally disconnected field of characteristic 0 , with $\pi(\Gamma)$ Zariski dense, satisfies $\overline{\pi(\Gamma)}$ is compact.

The proof we present is in the spirit of the proof we gave of Theorem 6.5 so as to be generalizable to cocycles defined on general ergodic $G$-spaces. We expect these generalized results to be of use in describing "arithmetic" features of an ergodic action, but we do not discuss this here.

Proof. (i) The proof we gave of Theorem 6.5 can be applied if we can find a measurable $\Gamma$-map $\varphi: G / P \rightarrow H / H_{0}$ where $H_{0}$ is an algebraic subgroup of $H$ such that $\bigcap_{h \in H} h H_{0} h^{-1}=\{e\}$. As in Theorem 6.5, we can let $P^{\prime} \subset H$ be a minimal parabolic subgroup, use amenability to find a $\Gamma$-map $\varphi: G / P \rightarrow$ $M\left(H / P^{\prime}\right)$ and prove that each orbit in $M\left(H / P^{\prime}\right)$ under $H$ is locally closed. Again, as in 6.5, we can then assume $\varphi: G / P \rightarrow H / H_{1}$ where $H_{1}$ is the stabilizer of a measure in $M\left(H / P^{\prime}\right)$. Unlike the real case however, this stabilizer need not be algebraic. For example, the group may be compact which in the real case implies that it is the real points of an algebraic group, while in the complex case, of course, a compact group will not be algebraic. However, we can suppose $H$ is rationally represented on a finite dimensional complex space in such a way that $P^{\prime}$ is the stabilizer of a point in projective space. Let $\mu$ be the measure on $H / P^{\prime}$ stabilized by $H_{1}$. If $H_{1}$ is not compact, then using an argument as in Furstenberg's lemma (Lemma 5.9) we see that $\mu$ must be supported on the intersection of $H / P^{\prime}$ with the union of two proper projective subspaces. Choose a proper subspace $V$ so that $\mu\left(H / P^{\prime} \cap[V]\right)>0$, and $V$ has minimal dimension among all subspaces with this property. By the minimality property of $[\mathrm{V}]$ and $H_{1}$-invariance of $\mu$, the $H_{1}$-orbit of [ $[\mathrm{V}]$ must clearly be a finite union of projective subspaces. Hence, if we let $H_{0}$ be the Zariski closure of $H_{1}$, then $H_{0} \subset H$ is a proper algebraic subgroup. Since $H$ is simple and with trivial center $\cap h H_{0} h^{-1}=\{e\}$, and as we remarked at the beginning of the proof, this suffices.

We must now consider the case in which $H_{1}$ is compact. We then have a $\Gamma$-map $\varphi: G / P \rightarrow H / H_{1}$, so that if we let $\nu=\varphi_{*}(\mu), \nu$ is a quasi-invariant ergodic measure for the action of $\Gamma$ on $H / H_{1}$. (Unlike the previous paragraph, $\mu$ is now the natural measure class on $G / P$.) Consider the $\Gamma$-map $\varphi \times \varphi$ : $G / P \times G / P \rightarrow H / H_{1} \times H / H_{1}$. It is well known that on $G / P$, the $P$-action is essentially transitive, the conull orbit having $P \cap \bar{P}$ as stabilizer, where $\bar{P}$ is the opposite parabolic to $P$. Thus as a $G$-space, $G / P \times G / P$ will be essentially transitive with stabilizer $P \cap \bar{P}$, which is noncompact. By Moore's ergodicity 
theorem (§2), $\Gamma$ is therefore ergodic on $G / P \times G / P$. It follows that $\Gamma$ must also be ergodic on $\left(H / H_{1}, \nu\right) \times\left(H / H_{1}, \nu\right)$. Since $H_{1}$ is compact, the $H$-orbits on $H / H_{1} \times H / H_{1}$ are closed. Since $(\nu \times \nu)$ is ergodic under $\Gamma$, and $\Gamma$-orbits are of course contained in $H$-orbits, smoothness of the $H$-action on $H / H_{1} \times$ $H / H_{1}$ implies that $\nu \times \nu$ must be supported on an $H$-orbit. From Fubini's theorem, one easily deduces that $\nu$ must be supported on an $H_{2}$ orbit in $H / H_{1}$ where $H_{2}$ is a conjugate of $H_{1}$, and in particular is compact. Thus, support $(\nu)$ is compact. Since $\nu$ is quasi-invariant under $\pi(\Gamma)$, support $(\nu)$ is $\pi(\Gamma)$-invariant, and since $H_{1}$ is also compact, it follows that $\pi(\Gamma)$ is contained in a compact set. This completes the proof of (i).

(ii) Let $P^{\prime} \subset H$ be a minimal parabolic $K$-subgroup, so that $H_{K} / P_{K}^{\prime}$ is compact, and $P_{K}^{\prime}$ contains no normal algebraic subgroup. We again wish to apply the same type of argument as in the proof of Theorem 6.5. The first step is to prove the analogue of Theorem 5.7 over $K$. In fact the proof in [52] shows that $\mathrm{GL}(n, K)$ acts smoothly on $M\left(\mathbf{P}^{n-1}(K)\right)$. We can assume that we have a faithful rational representation of $H_{K}$ on $K^{n}$ so that $H_{K} / P_{K}^{\prime}$ is an orbit in $\mathbf{P}^{n-1}(K)$. By amenability of the $\Gamma$-action on $G / P$, there is a $\Gamma$-map $\varphi$ : $G / P \rightarrow M\left(H_{K} / P_{K}^{\prime}\right) \subset M\left(\mathbf{P}^{n-1}(K)\right)$. By smoothness of the $\operatorname{GL}(n, K)$-action on $M\left(\mathbf{P}^{n-1}(K)\right)$, we can view $\varphi$ as a map $\varphi: G / P \rightarrow[\mu \cdot \operatorname{GL}(n, K)] \cap$ $M\left(H_{K} / P_{K}^{\prime}\right)$ where $\mu \in M\left(H_{K} / P_{K}^{\prime}\right)$. Let $S$ be the stabilizer of $\mu$ in $\operatorname{GL}(n, K)$. If $S$ is compact in $\operatorname{PGL}(n, K)$, then by the argument in part (i), $\pi(\Gamma)$ will be compact in $\operatorname{PGL}(n, K)$, and so $\overline{\pi(\Gamma)}$ will also be compact in $H$. If not, then using an argument as in Furstenberg's lemma (Lemma 5.9), we can, as in part (i), assume the Zariski closure $L$ of $S$ is a proper algebraic subgroup. Furthermore, we can clearly assume from the construction of $L$ as in part (i), that for any $g \in \operatorname{GL}(n, K), \operatorname{dim}\left(H \cap g L g^{-1}\right)<\operatorname{dim} H$. By the condition of Zariski density, it therefore suffices to see that $\pi(\Gamma) \subset g L_{K} g^{-1}$ for some $g \in \operatorname{GL}(n, K)$. We have $\varphi: G / P \rightarrow \operatorname{GL}(n, K) / L_{K}$ a measurable $\Gamma$-map. In the real case we showed $\varphi$ was rational by showing it could be built from homomorphisms of unipotent subgroups of $G$ which had to be rational. In the present situation, we can construct the same type of homomorphism using the argument of Theorem 6.5 , but now, since the image group is totally disconnected, these maps must be constant. We thus conclude that $\varphi: G / P \rightarrow \operatorname{GL}(n, K) / L_{K}$ is essentially constant. Since $\pi(\Gamma)$ leaves $\varphi(G / P)$ fixed, this implies $\pi(\Gamma)$ is contained in a $\mathrm{GL}(n, K)$-conjugate of $L_{K}$, and this completes the proof.

We now turn to the proof of Theorem 9.6 itself. We may take the semisimple Lie group to be $G_{\mathbf{R}}^{0}$, where now $G \subset \operatorname{GL}(n, \mathbf{C})$ represents an algebraic group defined over $\mathbf{Q}$. Thus $\Gamma \subset G_{\mathbf{R}}^{0}$ is an irreducible lattice. The following lemma is classical, and follows for example from an argument of Selberg [42] (see also [40, Proposition 6.6] for the same argument). This argument is based on expressing the embeddings of $\Gamma$ into $G$ as an algebraic variety, and then choosing a real algebraic point of this variety. However, with Theorem 9.7 at hand, we present an alternative argument due to Margulis [29].

LEMMA 9.8. There is a real algebraic number field $k$ and a rational faithful representation of $G$ such that, identifying $G$ with its image under this representation, $\Gamma \subset G_{k}$. 
Proof. The first step is to show that for $K$ the field of real algebraic numbers we have $\operatorname{Tr}(\operatorname{Ad}(\gamma)) \in K$ for all $\gamma$. Following Margulis, we let $\sigma$ be an automorphism of $\mathbf{C}$. Then $\sigma$ acts on matrices with entries in $\mathbf{C}$ by taking $\left(z_{i j}\right) \rightarrow\left(\sigma\left(z_{i j}\right)\right)$, and since $G$ is defined over $\mathbf{Q}, \boldsymbol{\sigma}$ induces an automorphism of $G$. (Of course this is an automorphism of $G$ only as an abstract group, and will in general not be measurable.) Let us assume for the moment that $G$ is simple. Then $\sigma \mid \Gamma$ satisfies either (i) $\overline{\sigma(\Gamma)}$ is compact; or (ii) $\sigma \mid \Gamma$ extends to a rational automorphism of $G$. This follows from Theorem 9.7. In the first case, all eigenvalues of $\operatorname{Ad}(\sigma(\gamma))$ will have absolute value one, and in the second case, these eigenvalues coincide with those of $\operatorname{Ad}(\gamma)$. (We remark that if $A: G \rightarrow G$ is an automorphism we have $d A \circ \operatorname{Ad}(A(g)) \circ(d A)^{-1}=\operatorname{Ad}(g)$, so $\operatorname{Tr}(\operatorname{Ad} A(g)$ ) $=\operatorname{Tr}(\operatorname{Ad} g)$.) Hence for each $\gamma \in \Gamma,\{\sigma(\operatorname{Tr}(\operatorname{Ad}(\gamma))) \mid \sigma \in \operatorname{Aut}(\mathbf{C})\}$ is bounded. The same can easily be seen if $G$ is semisimple by examining the composition of $\boldsymbol{\sigma} \mid \Gamma$ with projection on the simple factors. However, since $\operatorname{Aut}(\mathbf{C})$ is transitive on the transcendental numbers, it follows that $\operatorname{Tr}(\operatorname{Ad}(\gamma))$ is algebraic for all $\gamma \in \Gamma$.

Thus, identifying $G$ with $\operatorname{Ad}(G)$, we have $\Gamma \subset G$ with $\operatorname{Tr}(\gamma) \in K$ for all $\gamma \in \Gamma$. The next step, which is classical, is to observe that this implies that there is a faithful rational representation of $G$, defined over $K$, such that $\Gamma \subset G_{K}$, once again, identifying $G$ with its image under this representation. We recall the construction. Consider $\operatorname{Tr}: G \rightarrow \mathbf{C}$, and let $V=\mathbf{C}$-linear span of $\Gamma$-translates of $\operatorname{Tr}$. By the Borel density theorem $V$ will also be $G$-invariant. Choose a basis of $V$ of the form $\gamma_{i}$. Tr. Then one can verify in a straightforward manner that with respect to this basis, the matrix elements of $\gamma \in \Gamma$ acting on this space are all in $K$. Since $\Gamma$ is finitely generated (in the property (T) case this follows easily [9]) we can find an algebraic number field $k$ with $\Gamma \subset G_{k}$.

We now recall the basic operation of restriction of scalars. Suppose $G$ is an algebraic group defined over an algebraic number field $k$. Then there exists an algebraic group $\tilde{G}$ defined over $\mathbf{Q}$ such that

(i) there is an injective map $\alpha: G_{k} \rightarrow \tilde{G}_{\mathbf{Q}}$; and

(ii) there is a surjective rational homomorphism $p: \tilde{G} \rightarrow G$ defined over $k$ such that $p\left(\tilde{G}_{\mathbf{Q}}\right)=G_{k}$ and $p \circ \alpha: G_{k} \rightarrow G_{k}$ is the identity.

We recall here two ways of describing this construction. We can take $\tilde{G}=\prod_{\sigma} \sigma(G)$ where $\sigma$ runs through the distinct embeddings of $k$ in $\mathbf{C}$. Then $\alpha$ : $G_{k} \rightarrow \tilde{G}$ is the map $\alpha(g)=\left(\sigma_{1}(g), \ldots, \sigma_{r}(g)\right)$, and $p: \tilde{G} \rightarrow G$ is projection onto the factor corresponding to $\sigma=\mathrm{id}$. Alternatively, let $[k: Q]=r$, and choose an identification $k^{n} \leftrightarrow \mathbf{Q}^{n r}$. Then we have a map $\alpha: G_{k} \rightarrow \operatorname{GL}(n r, \mathbf{Q})$, and we let $\tilde{G}$ be the Zariski closure of $\alpha\left(G_{k}\right)$ in $\mathrm{GL}(n r, \mathbf{C})$. In this formulation, $p$ arises from the fact that the entries of $g \in G_{k}$ are described by $k$-linear combinations of the entries of $\alpha(g)$. These linear expressions allow us to define a linear map from $n r \times n r$ Q-matrices to $n \times n k$-matrices, and thus a map $\tilde{G} \rightarrow G$. (Recall that $G$ is the Zariski closure of $G_{k}$.) Since this map is clearly a homomorphism on $\alpha\left(G_{k}\right)$ (being the inverse of $\alpha$ ), it is also a homomorphism on its Zariskiclosure, $\tilde{G}$.

Completion of Proof of Theorem 9.6. We let $k$ be as in Lemma 9.8, and $\tilde{G}, p, \alpha$ as above. We have $\Gamma \subset G_{k}$, and we let $H \subset \tilde{G}$ be the Zariski closure of 
$\Gamma$. We still have $p(H)=G$ by Zariski density of $\Gamma$ in $G$. Since $G$ is semisimple, $p$ trivial on the radical of $H$, and, replacing $H$ by the quotient of $H$ by its radical, we can assume $H$ is a semisimple group defined over $\mathbf{Q}$, and with trivial center.

We now claim that $(\operatorname{ker} p)_{\mathbf{R}}$ is compact. Let $F$ be a simple factor of ker $p$. Then as algebraic groups defined over $\mathbf{R}$, we can write $H \cong G \times F \times F^{\prime}$ where $F^{\prime}$ is the product of the remaining simple factors. Since $\alpha(\Gamma)$ is Zariski dense in $H,(q \circ \alpha)(\Gamma)$ is Zariski dense in $F$ where $q$ is projection of $H$ onto $F$. We claim $F_{\mathbf{R}}$ must be compact. If not, then $(q \circ \alpha)(\Gamma)$ cannot have compact (topological) closure since compact real matrix groups are real points of algebraic groups. This would imply by Theorem 97 that $q \circ \alpha$ extended to a rational homomorphism $h: G \rightarrow F$. But then $\left\{\left(g, h(g), f^{\prime}\right) \mid g \in G, f^{\prime} \in F^{\prime}\right\}$ would be a proper algebraic subgroup containing $\alpha(\Gamma)$, contradicting Zariski density of $\alpha(\Gamma)$ in $H$. This verifies compactness of $F_{\mathbf{R}}$, and doing the same for each factor, compactness of $(\operatorname{ker} p)_{\mathbf{R}}$.

Now consider $\alpha: \Gamma \rightarrow H_{\mathbf{Q}}$. For each prime $a$, the image of $\alpha(\Gamma)$ in $H_{\mathbf{Q}_{a}}$ must be bounded by Theorem 9.7(ii). This means that the powers of each prime appearing in the denominators of matrix entries of $\alpha(\gamma) \in H_{\mathbf{Q}}$ are bounded uniformly over $\gamma \in \Gamma$. But $\Gamma$ is finitely generated, and hence only finitely many primes will appear at all. This is readily seen to imply that $\alpha(\Gamma) \cap H_{Z}$ is of finite index in $\alpha(\Gamma)$, and hence, applying $p$, that $\Gamma \cap p\left(H_{Z}\right)$ is of finite index in $\Gamma$. This in turn implies that $\Gamma \cap p\left(H_{Z}\right)$ is a lattice in $G_{\mathbf{R}}$. By Theorem 9.1 and Proposition 9.4, $p\left(H_{Z}\right)$ is a lattice in $G_{\mathbf{R}}$, and since $\left(\Gamma \cap p\left(H_{Z}\right)\right) \subset p\left(H_{Z}\right)$ is an inclusion of lattices, we also have $\left[p\left(H_{Z}\right): \Gamma \cap p\left(H_{Z}\right)\right]<\infty$. This shows commensurability of $\Gamma$ and $p\left(H_{Z}\right)$, completing the proof.

\section{REFERENCES}

1. L. Auslander, L. Green and F. Hahn, Flows on homogeneous spaces, Ann. of Math. Studies, no. 53, Princeton Univ. Press, Princeton, N.J., 1963.

2. L. Auslander, An exposition of the structure of solvmanifolds, Bull. Amer. Math. Soc. 79 (1973), 227-285.

3. A. Borel, Linear algebraic groups, Benjamin, New York, 1969.

4. __ Density properties for certain subgroups of semisimple Lie groups without compact factors, Ann. of Math. 72 (1960), 179-188.

5. A. Borel and Harish-Chandra, Arithmetic subgroups of algebraic groups, Ann. of Math. 75 (1962), 485-535.

6. A. Borel and J. P. Serre, Théorèmes de finitude en cohomologie Galoisienne, Comment. Math. Helv. 39 (1964), 111-164.

7. J. Brezin and C. C. Moore, Flows on homogeneous spaces: A new look (preprint).

8. A. Connes, J. Feldman and B. Weiss, Amenable equivalence relations are generated by a single transformation (preprint).

9. C. Delaroche and A. Kirillov, Sur les relations entre l'espace dual d'un groupe et la structure de ses sous-groupes fermés, Seminarie Bourbaki, no. 343, 1967/68.

10. H. A. Dye, On groups of measure preserving transformations. I, Amer. J. Math. 81 (1959), $119-159$.

11. $551-576$

12. E. G. Effros, Transformation groups and $C^{*}$-algebras, Ann. of Math. 81 (1965), 38-55.

13. J. Feldman, P. Hahn and C. C. Moore, Orbit structure and countable sections for actions of continuous groups, Adv. in Math. 28 (1978), 186-230. 
14. H. Freudenthal, Topologische Gruppen mit Genugend Vielen Fastperiodishen Funktionen, Ann. of Math. 37 (1936), 57-77.

15. H. Furstenberg, A Poisson formula for semisimple Lie groups, Ann. of Math. 77 (1963), $335-383$.

16. __ The structure of distal flows, Amer. J. Math. 85 (1963), 477-515.

17. Ergodic behavior of diagonal measures and a theorem of Szemeredi on arithmetic progressions, J. Analyse Math. 31 (1977), 204-256.

18. F. P. Greenleaf, Invariant means on topological groups, Van Nostrand, New York, 1969.

19. Y. Guivarc'h, Croissance polynomiale et periodes des fonctions harmonique, Bull. Soc. Math. France 101 (1973), 333-379.

20. G. Hedlund, The dynamics of geodesic flows, Bull. Amer. Math. Soc. 45 (1939), 241-260.

21. E. Hopf, Statistik der Lösungen geodätischer probleme vom unstabilen typus, Math. Ann. 117 (1940), 590-608.

22. R. Howe and C. C. Moore, Asymptotic properties of unitary representations, J. Funct. Anal. 32 (1979), 72-96.

23. J. W. Jenkins, Growth of connected locally compact groups, J. Funct. Anal. 12 (1973), 113-127.

24. D. Kazhdan, Connection of the dual space of a group with the structure of its closed subgroups, Functional Anal. Appl. 1 (1967), 63-65.

25. W. Krieger, On ergodic flows and the isomorphism of factors, Math. Ann. 223 (1976), 19-70.

26. G. W. Mackey, Ergodic transformation groups with a pure point spectrum, Illinois J. Math. 8 (1964), 593-600.

27. G. A. Margulis, Non-uniform lattices in semisimple algebraic groups, in Lie groups and their representations (I. M. Gelfand, ed.), Wiley, New York.

28. Discrete groups of motions of manifolds of nonpositive curvature, Amer. Math. Soc. Transl., vol. 109 (1977), 33-45.

29. __ Arithmeticity of irreducible lattices in semisimple groups of rank greater than 1, Appendix to Russian translation of M. Ragunathan, Discrete subgroups of Lie groups, Mir, Moscow, 1977. (Russian)

30. ___ Factor groups of discrete subgroups, Soviet Math. Dokl. 19 (1978), 1145-1149.

31. __ Quotient groups of discrete subgroups and measure theory, Functional Anal. Appl. 12 (1978), 295-305.

32. C. C. Moore, Ergodicity of flows on homogeneous spaces, Amer. J. Math. 88 (1966), 154-178.

33. __ Amenable subgroups of semisimple groups and proximal flows, Israel J. Math. 34 (1979), 121-138.

34. C. C. Moore and R. J. Zimmer, Groups admitting ergodic actions with generalized discrete spectrum, Invent. Math. 51 (1979), 171-188.

35. G. D. Mostow, Quasi-conformal mappings in n-space and the rigidity of hyperbolic space forms, Publ. Math. I.H.E.S. (1967), 53-104.

36. Strong rigidity of locally symmetric spaces, Ann. of Math. Studies, no. 78, Princeton Univ. Press, Princeton, N.J., 1973.

37. D. Ornstein and B. Weiss (to appear).

38. W. Parry, Zero entropy of distal and related transformations, Topological Dynamics (J. Auslander and W. Gottschalk, eds.), Benjamin, New Yor, 1968.

39. G. Prasad, Strong rigidity of Q-rank 1 lattices, Invent. Math. 21 (1973), 255-286.

40. M. Ragunathan, Discrete subgroups of Lie groups, Springer-Verlag, New York, 1972.

41. A. Ramsey, Virtual groups and group actions, Adv. in Math. 6 (1971), 253-322.

42. A. Selberg, On discontinuous groups in higher dimensional symmetric spaces, Int. Colloquium on Function Theory, Tata Institute, Bombay, 1960.

43. T. Sherman, $A$ weight theory for unitary representations, Canad. J. Math. 18 (1966), 159-168.

44. C. Sutherland, Orbit equivalence: Lectures on Krieger's theorem, Univ. of Oslo Lecture Notes.

45. V. S. Varadarajan, Geometry of quantum theory. II, Van Nostrand, Princeton, N.J., 1970.

46. S. P. Wang, On isolated points in the dual spaces of locally compact groups, Math. Ann. 218 (1975), 19-34.

47. R. J. Zimmer, Extensions of ergodic group actions, Illinois J. Math. 20 (1976), 373-409.

48. __ Ergodic actions with generalized discrete spectrum, Illinois J. Math. 20 (1976), $555-588$ 
49. Orbit spaces of unitary representations, ergodic theory, and simple Lie groups, Ann. of Math. 106 (1977), 573-588.

50. Uniform subgroups and ergodic actions of exponential Lie groups, Pacific J. Math. 78 (1978), 267-272.

51. Amenable ergodic group actions and an application to Poisson boundaries of random walks, J. Funct. Anal. 27 (1978), 350-372.

52. __ Induced and amenable ergodic actions of Lie groups, Ann. Sci. École Norm. Sup. (4) 11 (1978), 407-428.

53. __ Algebraic topology of ergodic Lie group action and measurable foliations (preprint).

54. __ An algebraic group associated to an ergodic diffeomorphism, Compositio Math. 43 (1981), 59-69.

55. Strong rigidity for ergodic actions of semisimple Lie groups, Ann. of Math. 112 (1980), 511-529.

56. _ Orbit equivalence and rigidity of ergodic actions of Lie groups, Ergod. Th. and Dynam. Sys. 1 (1981), 237-253.

57. On the cohomology of ergodic actions of semisimple Lie groups and discrete subgroups, Amer. J. Math. 103 (1981), 937-950.

58. __ On the Mostow rigidity theorem and measurable foliations by hyperbolic space (preprint).

Department of Mathematics, University of Chicago, Chicago, Illinois 60637 